\title{
On Henig Regularization of Material Design Problems for Quasi-Linear p-Biharmonic Equation
}

\author{
Peter Kogut ${ }^{1}$, Günter Leugering², Ralph Schiel ${ }^{2}$ \\ ${ }^{1}$ Department of Differential Equations, Dnipropetrovsk National University, Dnipro, Ukraine \\ ${ }^{2}$ Department Mathematik, Lehrstuhl II Universität Erlangen-Nürnberg Cauerstr, Erlangen, Germany \\ Email:p.kogut@i.ua, guenter.leugering@fau.de,ralph.schiel@fau.de
}

Received 25 June 2016; accepted 21 August 2016; published 24 August 2016

Copyright $@ 2016$ by authors and Scientific Research Publishing Inc.

This work is licensed under the Creative Commons Attribution International License (CC BY). http://creativecommons.org/licenses/by/4.0/

(c) (1) Open Access

\section{Abstract}

We study a Dirichlet optimal design problem for a quasi-linear monotone $p$-biharmonic equation with control and state constraints. We take the coefficient of the $p$-biharmonic operator as a design variable in $B V(\Omega)$. In this article, we discuss the relaxation of such problem.

\section{Keywords}

\section{p-Biharmonic Problem, Optimal Design, Relaxation, Henig Dilating Cone, Existence Result}

\section{Introduction}

The aim of this article is to analyze the following optimal design problem (OCP), which can be regarded as an optimal control problem, for quasi-linear partial differential equation (PDE) with mixed boundary conditions

$$
\text { Minimize }\left\{I(u, y)=\int_{\Omega}\left|y-y_{d}\right|^{2} \mathrm{~d} x+\int_{\Omega}|D u|\right\}
$$

subject to the quasi-linear equation

$$
\begin{aligned}
& \Delta\left(u|\Delta y|^{p-2} \Delta y\right)+F(x, y)=f \quad \text { in } \Omega, \\
& y=\frac{\partial y}{\partial v}=0 \text { on } \Gamma_{D}, \quad y=\Delta y=0 \text { on } \Gamma_{S},
\end{aligned}
$$

the pointwise state constraints 


$$
0 \leq \frac{\partial y(s)}{\partial v} \leq \zeta^{\max }(s) \text { a.e. on } \Gamma_{s}
$$

and the design (control) constraints

$$
u \in B V(\Omega) \text { and } \xi_{1}(x) \leq u(x) \leq \xi_{2}(x) \text { a.e. in } \Omega \text {. }
$$

Here, $\Gamma_{D}$ and $\Gamma_{S}$ are the disjoint part of the boundary $\partial \Omega\left(\partial \Omega=\Gamma_{D} \cup \Gamma_{S}\right), B V(\Omega)$ stands for the control space, $y_{d}, f$, and $\zeta^{\max }$ are given distributions. Problems of this type appear for $p$-power-like elastic isotropic flat plates of uniform thickness, where the design variable $u$ is to be chosen such that the deflection of the plate matches a given profile. The model extends the classical weighted biharmonic equation, where the weight $u=a^{3}$ involves the thickness $a$ of the plate, see e.g. [1]-[3], or $u$ can be regarded as a rigidity parameter. The OCP (1)-(4) can be considered as a prototype of design problems for quasilinear state equations. For an interesting exposure to this subject we can refer to the monographs [4]-[6].

A particular feature of OCP (1)-(4) is the restriction by the pointwise constraints (4) in $L^{p}\left(\Gamma_{S}\right)$-space. In fact, the ordering cone of positive elements in $L^{p}$-spaces is typically non-solid, i.e. it has an empty topological interior. Following the standard multiplier rule, which gives a necessary optimality condition for local solutions to state constrained OCPs, the constraint qualifications such as the Slater condition or the Robinson condition should be applied in this case. However, these conditions cannot be verified for cones such as

$L_{+}^{p}\left(\Gamma_{S}\right)=\left\{v \in L^{p}\left(\Gamma_{S}\right) \mid v \geq 0\right.$ a.e. in $\left.\Omega\right\}$ due to the fact that $\operatorname{int}\left(L_{+}^{p}\left(\Gamma_{S}\right)\right)=\varnothing$, where $\operatorname{int}(A)$ stands for the topological interior of the set A. Therefore, our main intention in this article is to propose a suitable relaxation of the pointwise state constraints in the form of some inequality conditions involving a so-called Henig approximation $\left(L_{+}^{p}\left(\Gamma_{S}\right)\right)_{S_{c}}(B)$ of the ordering cone of positive elements $L_{+}^{p}\left(\Gamma_{S}\right)$. Here, $B$ is a fixed closed base of $L_{+}^{p}\left(\Gamma_{S}\right)$. Due to fact that $L_{+}^{p}\left(\Gamma_{S}\right) \subset\left(L_{+}^{p}\left(\Gamma_{S}\right)\right)(B)$ for all $\varepsilon>0$, we can replace the cone $L_{+}^{p}\left(\Gamma_{S}\right)$ by its approximation $\left(L_{+}^{p}\left(\Gamma_{S}\right)\right)_{\varepsilon}(B)$. As a result, it leads to some relaxation of the inequality constraints of the considered problem, and, hence, to the approximation of the feasible set to the original OCP. Hence, the solvability of a given class of OCPs can be characterized by solving the corresponding Henig relaxed problems in the limit $\varepsilon \rightarrow 0$.

As was shown in the recent publication [7], the proposed approach is numerically viable for state-constrained optimal control problems with the state equation given by linear partial differential equations. In particular, using the finite element discretization of the Henig dilating cone of positive functions, it has been shown in [7] that the above approximation scheme, called conical regularization, where the regularization is done by replacing the ordering cone with a family of dilating cones, leads to a finite-dimensional optimization problem which can conveniently be treated by known numerical techniques. The non-emptiness of the feasible set for the stateconstrained OCPs is an open question even for the simplest situation. Therefore, we consider a more flexible notion of solution to the boundary value problem (2)-(3). With that in mind we discuss a variant of the penalization approach, called the "variational inequality (VI) method". Following this approach we weaken the requirements on admissible solutions to the original OCP and consider instead the family of penalized OCPs for appropriate variational inequalities

$$
\left\langle\Delta\left(u|\Delta y|^{p-2} \Delta y\right)+F(x, y), \zeta-y\right\rangle_{\left(\mathbb{W}_{p}(\Omega)\right)^{*} ; \mathbb{W}_{p}(\Omega)} \geq\langle f, \zeta-y\rangle_{\left(\mathbb{W}_{p}(\Omega)\right)^{*} ; \mathbb{W}_{p}(\Omega)}, \quad \forall \zeta \in K_{\varepsilon},
$$

where the sets $K_{\varepsilon}$ are defined in a special way. As a result, we show that each of new penalized OCP is solvable for each $\varepsilon>0$ and their solutions can be used for approximation of optimal pairs to the original problem.

The outline of the paper is the following. In Section 2 we report some preliminaries and notation we need in the sequel. In Sections 3, we give a precise statement of the state constrained optimal control (or design) problem and describe the main assumptions on the initial data and control functions. In Section 4, we provide the results concerning solvability of the original problem with control and state constraints. We show that this problem admits at least one solution if and only if the corresponding set of feasible solutions is nonempty. In Section 5 we show that the pointwise state constraints can be replaced by the weakened conditions coming from Henig relaxation of ordering cones. As a result, we give a precise definition of the relaxed optimization problems and show that the solvability of the original OCP can be characterized by the associated relaxed problems. In particular, we prove that the optimal solution to the original problem can be attained in the limit by the optimal solution of the relaxed problem. We consider in Section 6 the "variational inequality method" as an approximation of the OCPs. Following this approach, we weaken the requirements on feasible solutions to the original OCP. In 
contrast to the Henig relaxation approach, the penalized optimal control problem for indicated variational inequality has a non-empty feasible set and this problem is always solvable. In conclusion, we show that some of the optimal solutions to the original problem can be attained in the limit by optimal solutions of the penalized problem. However, it is unknown whether the entire set of the optimal solutions can be attained in such way.

\section{Definitions and Basic Properties}

Let $\Omega$ be a bounded open connected subset of $\mathbb{R}^{N}(N \geq 2)$. We assume that the boundary $\partial \Omega$ is Lipschitzian so that the unit outward normal $v=v(x)$ is well-defined for a.e. $x \in \partial \Omega$, where the abbreviation 'a.e.' should be interpreted here with respect to the $(N-1)$-dimensional Hausdorff measure. We also assume that the boundary $\partial \Omega$ consists of two disjoint parts $\partial \Omega=\Gamma_{D} \cup \Gamma_{S}$, where the sets $\Gamma_{D}$ and $\Gamma_{S}$ have positive $(N-1)$-dimensional measures, and $\Gamma_{S}$ is of $C^{2}$.

Let $p$ be a real number such that $2 \leq p<\infty$. By $W^{2, p}(\Omega)$ we denote the Sobolev space as the subspace of $L^{p}(\Omega)$ of functions $y$ having generalized derivatives $D^{k} y$ up to order $k=2$ in $L^{p}(\Omega)$. We note that thanks to interpolation theory, see ([8], Theorem 4.14), $W^{2, p}(\Omega)$ is a Banach space with respect to the norm

$$
\|y\|_{W^{2, p}(\Omega)}=\left(\|y\|_{L^{p}(\Omega)}^{p}+\left\|D^{2} y\right\|_{L^{p}(\Omega)}^{p}\right)^{1 / p}=\left(\int_{\Omega}\left(|y|^{p}+\left|D^{2} y\right|^{p}\right) \mathrm{d} x\right)^{1 / p},
$$

where

$$
\left|D^{2} y\right|=\left(D^{2} y \cdot D^{2} y\right)^{1 / 2}, \quad \text { and } \quad D^{2} y \cdot D^{2} v=\sum_{i_{1}, i_{2}=1}^{N} \frac{\partial^{2} y}{\partial x_{i_{1}} \partial x_{i_{2}}} \frac{\partial^{2} v}{\partial x_{i_{1}} \partial x_{i_{2}}} .
$$

For any $y \in C^{1}(\bar{\Omega})$ we define the traces

$$
\gamma_{0}(y)=\left.y\right|_{\partial \Omega} \text {, and } \gamma_{1}(y)=\left.\frac{\partial y}{\partial v}\right|_{\partial \Omega} .
$$

By ([9], Theorem 8.3), these linear operators can be extended continuously to the whole of space $W^{2, p}(\Omega)$. We set

$$
W^{2-1 / p, p}(\partial \Omega):=\gamma_{0}\left[W^{2, p}(\Omega)\right], \quad W^{1-1 / p, p}(\partial \Omega):=\gamma_{1}\left[W^{2, p}(\Omega)\right]
$$

as closed subspaces of $W^{1, p}(\partial \Omega)$ and $L^{p}(\partial \Omega)$, respectively. Moreover, the injections

$$
W^{2-1 / p, p}(\partial \Omega) \hookrightarrow W^{1, p}(\partial \Omega) \text { and } W^{1-1 / p, p}(\partial \Omega) \hookrightarrow L^{p}(\partial \Omega)
$$

are compact.

Let $C_{0}^{\infty}\left(\mathbb{R}^{N} ; \Gamma_{D}\right)=\left\{\varphi \in C_{0}^{\infty}\left(\mathbb{R}^{N}\right): \varphi=0\right.$ and $\frac{\partial \varphi}{\partial v}=0$ on $\left.\Gamma_{D}\right\}$. We define the Banach space $W_{0}^{2, p}\left(\Omega ; \Gamma_{D}\right)$ as the closure of $C_{0}^{\infty}\left(\mathbb{R}^{N} ; \Gamma_{D}\right)$ with respect to the norm $\|y\|_{W^{2, p}(\Omega)}$. Let $W^{-2, q}\left(\Omega ; \Gamma_{D}\right)$ be the dual space to $W_{0}^{2, p}\left(\Omega ; \Gamma_{D}\right)$, where $q=p /(p-1)$ is the conjugate of $p$. We also define the space $W_{0}^{1, p}(\Omega)$ as the closure of $C_{0}^{\infty}(\Omega)$ with respect to the norm $\|y\|_{W_{0}^{1, p}(\Omega)}=\left(\int_{\Omega}|\nabla y|^{p} \mathrm{~d} x\right)^{1 / p}$.

Throughout this paper, we use the notation $\mathbb{W}_{p}(\Omega):=W_{0}^{2, p}\left(\Omega ; \Gamma_{D}\right) \cap W_{0}^{1, p}(\Omega)$. Let us notice that $\mathbb{W}_{p}(\Omega)$ equipped with the norm

$$
\|y\|_{p, \Delta}:=\|\Delta y\|_{L^{p}(\Omega)}=\left(\int_{\Omega}|\Delta y|^{p} \mathrm{~d} x\right)^{1 / p}=\left(\int_{\Omega}\left|\sum_{i=1}^{N} \frac{\partial^{2} y}{\partial x_{i}^{2}}\right|^{p} \mathrm{~d} x\right)^{1 / p}
$$

is a uniformly convex Banach space [10]. Moreover, the norm $\|\cdot\|_{p, \Delta}$ is equivalent on $\mathbb{W}_{p}(\Omega)$ to the usual norm of $W^{2, p}(\Omega)$. Indeed, since the Laplace operator $-\Delta$ acts from $\mathbb{W}_{p}(\Omega)$ in $L^{p}(\Omega)$ and the Dirichlet boundary value problem

$$
\Delta y=f \text { in } \Omega, \quad y=0 \text { on } \partial \Omega
$$


is uniquely solvable in $\mathbb{W}_{p}(\Omega)$ for all $f \in L^{p}(\Omega)$, it follows that the inverse operator $T:=(-\Delta)^{-1}$ : $L^{p}(\Omega) \rightarrow W^{2, p}(\Omega) \cap W_{0}^{1, p}(\Omega)$ is well defined and satisfies the following elliptic regularity estimate [11]

$$
\|T f\|_{W^{2, p}(\Omega)} \leq C_{p}\|f\|_{L^{p}(\Omega)} .
$$

This allows us to conclude the following. If $f \in L^{p}(\Omega)$ and $y \in W_{0}^{1, p}(\Omega)$ are such that $\frac{\partial y}{\partial v}=0$ on $\Gamma_{D}$ and $y$ is a solution of (8), then $-\Delta y \in L^{p}(\Omega), y=0$ on the boundary $\partial \Omega$, and, therefore, $y \in \mathbb{W}_{p}(\Omega)$. Hence,

$$
\|y\|_{W^{2, p}(\Omega)}=\|T(-\Delta y)\|_{W^{2, p}(\Omega)} \leq C_{p}\|\Delta y\|_{L^{p}(\Omega)}=C_{p}\|y\|_{p, \Delta},
$$

for a suitable positive constant $C_{p}$ independent of $f$. On the other hand, it is easy to see that

$$
\|y\|_{p, \Delta} \leq\|y\|_{W^{2, p}(\Omega)} .
$$

Thus, by the Closed Graph Theorem, we can conclude that $\|\cdot\|_{p, \Delta}$ is equivalent to the norm induced by $W^{2, p}(\Omega)$ (for the details we refer to [12] [13]).

By $B V(\Omega)$ we denote the space of all functions in $L^{1}(\Omega)$ for which the norm

$$
\begin{aligned}
\|f\|_{B V(\Omega)} & =\|f\|_{L^{1}(\Omega)}+\int_{\Omega}|D f| \\
& =\|f\|_{L^{1}(\Omega)}+\sup \left\{\int_{\Omega} f \operatorname{div} \varphi \mathrm{d} x: \varphi \in C_{0}^{1}\left(\Omega ; \mathbb{R}^{N}\right),|\varphi(x)| \leq 1 \text { for } x \in \Omega\right\}
\end{aligned}
$$

is finite.

We recall that a sequence $\left\{f_{k}\right\}_{k=1}^{\infty}$ converges weakly-* to $f$ in $B V(\Omega)$ if and only if the two following conditions hold (see [14]): $f_{k} \rightarrow f$ strongly in $L^{1}(\Omega)$ and $D f_{k} \rightarrow D f$ weakly-* in the space of Radon measures $\mathcal{M}(\Omega)$, i.e.

$$
\lim _{k \rightarrow \infty} \int_{\Omega} \varphi D f_{k}=\int_{\Omega} \varphi D f \quad \forall \varphi \in C_{0}(\Omega) .
$$

It is well-known also the following compactness result for $B V$-spaces (Helly's selection theorem, see [15]).

Theorem 1. If $\left\{f_{k}\right\}_{k=1}^{\infty} \subset B V(\Omega)$ and $\sup _{k \in \mathbb{N}}\left\|f_{k}\right\|_{B V(\Omega)}<+\infty$, then there exists a subsequence of $\left\{f_{k}\right\}_{k=1}^{\infty}$ strongly converging in $L^{1}(\Omega)$ to some $f \in B V(\Omega)$ such that $D f_{k}{ }^{*} D f$ weakly-* in the space of Radon measures $\mathcal{M}(\Omega)$. Moreover, if $\left\{f_{k}\right\}_{k=1}^{\infty} \subset B V(\Omega)$ strongly converges to some $f$ in $L^{1}(\Omega)$ and satisfies $\sup _{k \in \mathbb{N}} \int_{\Omega}\left|D f_{k}\right|<+\infty$, then

$$
\begin{aligned}
& \text { (i) } f \in B V(\Omega) \text { and } \int_{\Omega}|D f| \leq \liminf _{k \rightarrow \infty} \int_{\Omega}\left|D f_{k}\right| \text {; } \\
& \text { (ii) } f_{k} \stackrel{*}{\rightarrow} f \text { in } B V(\Omega) .
\end{aligned}
$$

\section{Setting of the Optimal Control Problem}

Let $\xi_{1}, \quad \xi_{2}$ be fixed elements of $L^{\infty}(\Omega) \cap B V(\Omega)$ satisfying the conditions

$$
0<\alpha \leq \xi_{1}(x) \leq \xi_{2}(x) \text { a.e. in } \Omega \text {, }
$$

where $\alpha$ is a given positive value.

Let $F: \Omega \times \mathbb{R} \rightarrow \mathbb{R}$ be a nonlinear mapping such that $F$ is in the space $\operatorname{Car}(\Omega \times \mathbb{R})$ of Carathéodory functions on $\Omega \times \mathbb{R}$, i.e.

1) the function $F(x, \cdot)$ is continuous in $\mathbb{R}$ for almost all $x \in \Omega$;

2) the function $F(\cdot, y)$ is measurable for each $y \in \mathbb{R}$.

In addition, the following conditions of subcritical growth, monotonicity, and non-negativity are fulfilled:

$$
|F(x, \eta)| \leq C_{1}|\eta|^{r-1} \quad \text { for a.e. } x \in \Omega \text { and all } \eta \in \mathbb{R} \text {, }
$$




$$
\begin{aligned}
& \left(F(x, \eta)-F\left(x, \eta^{\prime}\right)\right)\left(\eta-\eta^{\prime}\right)>0 \text { for a.e. } x \in \Omega \text { and all } \eta, \eta^{\prime} \in \mathbb{R}, \eta \neq \eta^{\prime}, \\
& F(x, \eta) \eta \geq 0 \text { for a.e. } x \in \Omega \text { and all } \eta \in \mathbb{R} .
\end{aligned}
$$

for some $r \in\left(1, p^{*}\right)$, where

$$
p^{*}= \begin{cases}N p /(N-2 p), & p<N / 2 \\ +\infty, & p \geq N / 2\end{cases}
$$

is the critical exponent for the Sobolev imbedding $W^{2, p}(\Omega) \hookrightarrow L^{r}(\Omega)$, and $C_{1}>0$. In particular, conditions (13) - (14) imply that $F(x, \cdot)$ is monotonically increasing on $\mathbb{R}$ and $F(x, 0)=0$ for almost all $x \in \Omega$.

Let $f \in W^{-2, q}\left(\Omega ; \Gamma_{D}\right), \quad y_{d} \in L^{2}(\Omega)$, and $\zeta^{\max } \in L^{p}(\partial \Omega)$ be given distributions. The optimal control problem we consider in this paper is to minimize the discrepancy between $y_{d}$ and the solutions of the following state-constrained boundary valued problem

$$
\begin{aligned}
& \Delta_{p}^{2}(u, y)+F(x, y)=f \quad \text { in } \Omega, \\
& y=\frac{\partial y}{\partial v}=0 \quad \text { on } \Gamma_{D}, \quad y=\Delta y=0 \quad \text { on } \Gamma_{s}, \\
& 0 \leq \frac{\partial y(s)}{\partial v} \leq \zeta^{\max }(s) \quad \text { a.e. on } \Gamma_{s}
\end{aligned}
$$

by choosing an appropriate function $u \in \mathfrak{A}_{a d}$ as control. Here,

$$
\Delta_{p}^{2}(u, y):=\Delta\left(u|\Delta y|^{p-2} \Delta y\right)
$$

is the operator of fourth order called the generalized $p$-biharmonic operator, and the class of admissible controls $\mathfrak{A}_{\text {ad }}$ we define as follows

$$
\mathfrak{A}_{a d}=\left\{u \in B V(\Omega) \mid \xi_{1}(x) \leq u(x) \leq \xi_{2}(x) \text { a.e. in } \Omega\right\} .
$$

It is clear that $\mathfrak{A}_{\text {ad }}$ is a nonempty convex subset of $L^{1}(\Omega)$ with an empty topological interior.

More precisely, we are concerned with the following optimal control problem

$$
\begin{aligned}
& \text { Minimize }\left\{I(u, y)=\int_{\Omega}\left|y-y_{d}\right|^{2} \mathrm{~d} x+\int_{\Omega}|D u|\right\} \\
& \text { subject to the constraints (15)-(18). }
\end{aligned}
$$

Before we will discuss the question of existence of admissible pairs to the problem (19), we note that the function $F \in \operatorname{Car}(\Omega \times \mathbb{R})$ can be associated with operator $F: \mathbb{W}_{p}(\Omega) \rightarrow\left(\mathbb{W}_{p}(\Omega)\right)^{*}$ defined by the rule

$$
\langle F(y), v\rangle_{\left(\mathbb{W}_{p}(\Omega)\right)^{*} ; \mathbb{W}_{p}(\Omega)}=\int_{\Omega} F(x, y) v \mathrm{~d} x, \quad \forall v \in \mathbb{W}_{p}(\Omega) .
$$

Moreover, taking into account the growth condition (12) and the compactness of the Sobolev imbedding $W_{0}^{2, p}\left(\Omega ; \Gamma_{D}\right) \hookrightarrow L^{r}(\Omega)$ for $r<p^{*}$ it is easy to show that operator $F: \mathbb{W}_{p}(\Omega) \rightarrow\left(\mathbb{W}_{p}(\Omega)\right)^{*}$ is compact.

Definition 3.1. We say that an element $y \in \mathbb{W}_{p}(\Omega)$ is the weak solution (in the sense of Minty) to the boundary value problem (15) - (16), for a given admissible control $u \in \mathfrak{A}_{\text {ad }}$, if

$$
\begin{aligned}
& \int_{\Omega} u|\Delta \varphi|^{p-2} \Delta \varphi(\Delta \varphi-\Delta y) \mathrm{d} x+\langle F(\varphi), \varphi-y\rangle_{\left(\mathbb{W}_{p}(\Omega)\right)^{*} ; \mathbb{W}_{p}(\Omega)} \\
& \geq\langle f, \varphi-y\rangle_{W^{-2, q}\left(\Omega ; \Gamma_{D}\right) ; \mathbb{W}_{0}^{2, p}\left(\Omega ; \Gamma_{D}\right)}, \quad \forall \varphi \in C_{0}^{\infty}\left(\Omega ; \Gamma_{D}\right) .
\end{aligned}
$$

Remark 3.1. Since the set $C_{0}^{\infty}\left(\mathbb{R}^{N} ; \Gamma_{D}\right)$ is dense in $\mathbb{W}_{p}(\Omega)$, it follows that the element $\varphi=y+t w$ with an arbitrary $w \in \mathbb{W}_{p}(\Omega)$ and $t>0$ can be taken as a test function in (21). As a result, (21) implies that

$$
\begin{aligned}
& \int_{\Omega} u|\Delta y+t \Delta w|^{p-2}(\Delta y+t \Delta w) \Delta w \mathrm{~d} x+\int_{\Omega} F(x, y+t w) w \mathrm{~d} x \\
& \geq\langle f, w\rangle_{W^{-2, q}\left(\Omega ; \Gamma_{D}\right) ; \mathbb{W}_{0}^{2, p}\left(\Omega ; \Gamma_{D}\right)}, \quad \forall w \in \mathbb{W}_{p}(\Omega) .
\end{aligned}
$$


Passing to the limit as $t \rightarrow 0$ (because $F \in \operatorname{Car}(\Omega \times \mathbb{R})$ ), we get

$$
\int_{\Omega} u|\Delta y|^{p-2} \Delta y \Delta w \mathrm{~d} x+\int_{\Omega} F(x, y) w \mathrm{~d} x \geq\langle f, w\rangle_{W^{-2, q}\left(\Omega ; \Gamma_{D}\right) ; W_{0}^{2, p}\left(\Omega ; \Gamma_{D}\right)}, \quad \forall w \in \mathbb{W}_{p}(\Omega) .
$$

Hence,

$$
\int_{\Omega} u|\Delta y|^{p-2} \Delta y \Delta w \mathrm{~d} x+\int_{\Omega} F(x, y) w \mathrm{~d} x=\langle f, w\rangle_{W^{-2, q}\left(\Omega ; \Gamma_{D}\right) ; W_{0}^{2, p}\left(\Omega ; \Gamma_{D}\right)},
$$

and we arrive at the standard definition of weak solution to the boundary value problem (15)-(16). However, in order to avoid some mathematical difficulties, we will mainly use the Minty inequality in our further analysis. It is worth to note that having applied Green's formula twice to operator $\Delta\left(u|\Delta y|^{p-2} \Delta y\right)$ tested by $v \in C_{0}^{\infty}\left(\Omega ; \Gamma_{D}\right)$, we arrive at the identity

$$
\begin{aligned}
& \int_{\Omega} \Delta\left(u|\Delta y|^{p-2} \Delta y\right) v \mathrm{~d} x \\
& =-\int_{\Omega}\left(\nabla\left(u|\Delta y|^{p-2} \Delta y\right), \nabla v\right)_{\mathbb{R}^{N}} \mathrm{~d} x+\int_{\partial \Omega} \frac{\partial}{\partial v}\left(u|\Delta y|^{p-2} \Delta y\right) v \mathrm{~d} \mathcal{H}^{N-1} \\
& =\int_{\Omega} u|\Delta y|^{p-2} \Delta y \Delta v \mathrm{~d} x-\int_{\Gamma_{D}} u|\Delta y|^{p-2} \Delta y \frac{\partial v}{\partial v} \mathrm{~d} \mathcal{H}^{N-1}-\int_{\Gamma_{S}} u|\Delta y|^{p-2} \Delta y \frac{\partial v}{\partial v} \mathrm{~d} \mathcal{H}^{N-1} \\
& =\int_{\Omega} u|\Delta y|^{p-2} \Delta y \Delta v \mathrm{~d} x-\int_{\Gamma_{S}} u|\Delta y|^{p-2} \Delta y \frac{\partial v}{\partial v} \mathrm{~d} \mathcal{H}^{N-1} \quad \forall v \in C_{0}^{\infty}\left(\Omega ; \Gamma_{D}\right) .
\end{aligned}
$$

Hence, if $y$ as an element of $\mathbb{W}_{p}(\Omega):=W_{0}^{2, p}\left(\Omega ; \Gamma_{D}\right) \cap W_{0}^{1, p}(\Omega)$ is the weak solution of the boundary value problem (15) - (16) in the sense of Definition 3.1, then relations (15)-(16) are fulfilled as follows (for the details, we refer to ([16], Section 2.4.4) and ([4], Section 2.4.2))

$$
\left.\begin{array}{l}
\Delta_{p}^{2}(u, y)+F(y)=f \quad \text { in }\left(C_{0}^{\infty}\left(\Omega ; \Gamma_{D}\right)\right)^{*}, \\
\gamma_{0}(y)=0 \text { in } W^{2-1 / p, p}(\partial \Omega), \\
\gamma_{1}(y)=0 \text { in } W^{1 / q, p}\left(\Gamma_{D}\right), \\
\gamma_{0}(\Delta y)=0 \text { in } W^{-1 / p, p}\left(\Gamma_{S}\right):=\left(W^{1 / p, q}\left(\Gamma_{S}\right)\right)^{*} .
\end{array}\right\}
$$

In particular, taking $w=y$ in (22), this yields the relation

$$
\int_{\Omega} u|\Delta y|^{p} \mathrm{~d} x+\int_{\Omega} F(x, y) y \mathrm{~d} x=\langle f, y\rangle_{W^{-2, q}\left(\Omega ; \Gamma_{D}\right) ; W_{0}^{2, p}\left(\Omega ; \Gamma_{D}\right)} .
$$

As a result, conditions (11), (18), and inequalities (14) and (9) lead us to the following a priori estimate

$$
\|y\|_{p, \Delta}:=\left(\int_{\Omega}|\Delta y|^{p} \mathrm{~d} x\right)^{1 / p} \leq\left(\alpha^{-1} C_{p}\|f\|_{W^{-2, q}\left(\Omega ; \Gamma_{D}\right)}\right)^{\frac{1}{p-1}} \quad \forall u \in \mathfrak{A}_{a d} .
$$

The existence of a unique weak solution to the boundary value problem (15)-(16) in the sense of Definition 3.1 follows from an abstract theorem on monotone operators.

Theorem 2 ([17]) Let $V$ be a reflexive separable Banach space. Let $V^{*}$ be the dual space, and let $A: V \rightarrow V^{*}$ be a bounded, hemicontinuous, coercive and strictly monotone operator. Then the equation Ay=f has a unique solution for each $f \in V^{*}$.

Here, the above mentioned properties of the strict monotonicity, hemicontinuity, and coercivity of the operator $A$ have respectively the following meaning:

$$
\begin{aligned}
& \langle A y-A v, y-v\rangle_{V^{*} ; V} \geq 0, \quad \forall y, v \in V ; \\
& \langle A y-A v, y-v\rangle_{V^{*} ; V}=0 \Rightarrow y=v ; \\
& \text { the function } t \mapsto\langle A(y+t v), w\rangle_{V^{*} ; V} \text { is continuous for all } y, v, w \in V ; \\
& \lim _{\|y\|_{V} \rightarrow \infty} \frac{\langle A y, y\rangle_{V^{*} ; V}}{\|y\|_{V}}=+\infty .
\end{aligned}
$$


In our case, we can define the operator $A(u, \cdot)$ as a mapping $\mathbb{W}_{p}(\Omega) \rightarrow\left(\mathbb{W}_{p}(\Omega)\right)^{*}$ by

$$
\langle A(u, y), w\rangle_{\left(\mathbb{W}_{p}(\Omega)\right)^{*} ; \mathbb{W}_{p}(\Omega)}:=\int_{\Omega} u|\Delta y|^{p-2} \Delta y \Delta w \mathrm{~d} x+\int_{\Omega} F(x, y) w \mathrm{~d} x .
$$

In view of the properties (12)-(14) and compactness of the Sobolev imbedding $W_{0}^{2, p}\left(\Omega ; \Gamma_{D}\right) \rightarrow L^{r}(\Omega)$ for $r<p^{*}$, it is easy to show that $A(u, y)=\Delta_{p}^{2}(u, y)+F(y)$ and $A(u, \cdot)$ satisfies all assumptions of Theorem 2 (for the details we refer to [16] [17]). Hence, the variational problem

$$
\begin{aligned}
& \text { For a given } u \in \mathfrak{A}_{a d} \text {, find } y \in \mathbb{W}_{p}(\Omega) \text { such that } \\
& \langle A(u, y), \varphi\rangle_{\left(\mathbb{W}_{p}(\Omega)\right)^{*} ; \mathbb{W}_{p}(\Omega)}=\langle f, \varphi\rangle_{\left(\mathbb{W}_{p}(\Omega)\right)^{*} ; \mathbb{W}_{p}(\Omega)}, \forall \varphi \in \mathbb{W}_{p}(\Omega)
\end{aligned}
$$

for which $A(u, y)=f$ is its operator form, has a unique solution $y=y(u) \in \mathbb{W}_{p}(\Omega)$. We note that the duality pairing in the right hand side of (30) makes a sense for any distribution $f \in W^{-2, q}\left(\Omega ; \Gamma_{D}\right)$ because $W^{-2, q}\left(\Omega ; \Gamma_{D}\right):=\left(W_{0}^{2, p}\left(\Omega ; \Gamma_{D}\right)\right)^{*} \subset\left(\mathbb{W}_{p}(\Omega)\right)^{*}$. It remains to show that the solution y of (30) satisfies the Minty relation (21). Indeed, in view of the monotonicity of $A$, we have

$$
\begin{aligned}
0 & \leq\langle A(u, v)-A(u, y), v-y\rangle_{\left(\mathbb{W}_{p}(\Omega)\right)^{*} ; \mathbb{W}_{p}(\Omega)} \\
& =\langle A(u, v), v-y\rangle_{\left(\mathbb{W}_{p}(\Omega)\right)^{*} ; \mathbb{W}_{p}(\Omega)}-\langle A(u, y), v-y\rangle_{\left(\mathbb{W}_{p}(\Omega)\right)^{*} ; \mathbb{W}_{p}(\Omega)} \\
& \quad \text { by (30) } \\
& =\langle A(u, v), v-y\rangle_{\left(\mathbb{W}_{p}(\Omega)\right)^{*} ; \mathbb{W}_{p}(\Omega)}-\langle f, \varphi\rangle_{\left(\mathbb{W}_{p}(\Omega)\right)^{*} ; \mathbb{W}_{p}(\Omega)} .
\end{aligned}
$$

Thus,

$$
\langle A(u, v), v-y\rangle_{\left(\mathbb{W}_{p}(\Omega)\right)^{*} ; \mathbb{W}_{p}(\Omega)} \geq\langle f, \varphi\rangle_{W^{-2, q}\left(\Omega ; \Gamma_{D}\right) ; \mathbb{W}_{0}^{2, p}\left(\Omega ; \Gamma_{D}\right)}, \quad \forall v \in \mathbb{W}_{p}(\Omega),
$$

and, hence, in view of Remark 3.1, the Minty relation (21) holds true.

Taking this fact into account, we adopt the following notion.

Definition 3.2. We say that $(u, y)$ is a feasible pair to the OCP (19) if $u \in \mathfrak{A}_{a d} \subset B V(\Omega), y \in \mathbb{W}_{p}(\Omega)$, the pair $(u, y)$ is related by the Minty inequality $(21), I(u, y)<+\infty$, and

$$
\gamma_{1}(y) \in L_{+}^{p}\left(\Gamma_{S}\right), \quad \zeta^{\max }-\gamma_{1}(y) \in L_{+}^{p}\left(\Gamma_{S}\right),
$$

where $L_{+}^{p}\left(\Gamma_{S}\right)$ stands for the natural ordering cone of positive elements in $L^{p}\left(\Gamma_{S}\right)$, i.e.

$$
L_{+}^{p}\left(\Gamma_{S}\right):=\left\{v \in L^{p}\left(\Gamma_{S}\right) \mid v \geq 0 \quad \mathcal{H}^{N-1} \text {-a.e. on } \Gamma_{S}\right\} .
$$

We denote by $\Xi$ the set of all feasible pairs for the OCP (19). We say that a pair $\left(u^{0}, y^{0}\right) \in B V^{1}(\Omega) \times \mathbb{W}_{p}(\Omega)$ is an optimal solution to problem (19) if

$$
\left(u^{0}, y^{0}\right) \in \Xi \quad \text { and } \quad I\left(u^{0}, y^{0}\right)=\inf _{(u, y) \in \Xi} I(u, y) .
$$

Remark 3.2. Before we proceed further, we need to make sure that minimization problem (19) is meaningful, i.e. there exists at least one pair $(u, y)$ such that $(u, y)$ satisfying the control and state constraints (16)-(18), $I(u, y)<+\infty$, and $(u, y)$ would be a physically relevant solution to the boundary value problem (15)-(16). In fact, one needs the feasible set $\Xi$ to be nonempty. But even if we are aware that $\Xi \neq \varnothing$, this set must be sufficiently rich in some sense, otherwise the OCP (19) becomes trivial. From a mathematical point of view, to deal directly with the control and especially state constraints is typically very difficult [18]-[20]. Thus, the nonemptiness of feasible set for OCPs with control and state constraints is an open question even for the simplest situation.

It is reasonably now to make use of the following Hypothesis.

$\left(H_{1}\right)$ There exists at least one pair $(u, y) \in B V(\Omega) \times \mathbb{W}_{p}(\Omega)$ such that $(u, y) \in \Xi$.

\section{Existence of Optimal Solutions}

In this section we focus on the solvability of optimal control problem (15)-(19). Hereinafter, we suppose that the 
space $B V(\Omega) \times \mathbb{W}_{p}(\Omega)$ is endowed with the norm $\|(u, y)\|_{B V(\Omega) \times \mathbb{W}_{p}(\Omega)}:=\|u\|_{B V(\Omega)}+\|y\|_{p, \Delta}$. Let $\tau$ be the topology on the set $\Xi \subset B V(\Omega) \times \mathbb{W}_{p}(\Omega)$ which we define as the product of the weak-* topology of $B V(\Omega)$ and the weak topology of $W_{0}^{2, p}\left(\Omega ; \Gamma_{D}\right)$.

We begin with a couple of auxiliary results.

Lemma 1. Let $\left\{\left(u_{k}, y_{k}\right) \in \Xi\right\}_{k \in \mathbb{N}}$ be a sequence such that $\left(u_{k}, y_{k}\right) \stackrel{\tau}{\longrightarrow}(u, y)$ in $B V(\Omega) \times \mathbb{W}_{p}(\Omega)$. Then we have

$$
\lim _{k \rightarrow \infty} \int_{\Omega} u_{k} \Delta y_{k} \Delta \varphi \mathrm{d} x=\int_{\Omega} u \Delta y \Delta \varphi \mathrm{d} x \quad \forall \varphi \in C_{0}^{\infty}\left(\Omega ; \Gamma_{D}\right) .
$$

Proof. Since $u_{k} \rightarrow u$ in $L^{1}(\Omega)$ and $\left\{u_{k}\right\}_{k \in \mathbb{N}}$ is bounded in $L^{\infty}(\Omega)$, we get that $u_{k} \rightarrow u$ strongly in $L^{r}(\Omega)$ for every $1 \leq r<+\infty$. In particular, we have that $u_{k} \rightarrow u$ in $L^{q}(\Omega)$ and $\Delta y_{k} \Delta \varphi \rightarrow \Delta y \Delta \varphi$ in $L^{p}(\Omega)$. Hence, it is immediate to pass to the limit and to deduce (33).

As a consequence, we have the following property.

Corollary 1. Let $\left\{\left(u_{k}, y_{k}\right) \in \Xi\right\}_{k \in \mathbb{N}}$ and $\left\{\zeta_{k} \in W_{0}^{2, q}\left(\Omega ; \Gamma_{D}\right)\right\}_{k \in \mathbb{N}}$ be sequences such that $\left(u_{k}, y_{k}\right) \stackrel{\tau}{\longrightarrow}(u, y)$ in $B V(\Omega) \times \mathbb{W}_{p}(\Omega)$ and $\zeta_{k} \rightarrow \zeta$ in $W_{0}^{2, q}\left(\Omega ; \Gamma_{D}\right)$. Then

$$
\lim _{k \rightarrow \infty} \int_{\Omega} u_{k} \Delta y_{k} \Delta \zeta_{k} \mathrm{~d} x=\int_{\Omega} u \Delta y \Delta \zeta \mathrm{d} x .
$$

Our next step concerns the study of topological properties of the feasible set $\Xi$ to problem (19).

The following result is crucial for our further analysis.

Theorem 3. Let $\left\{\left(u_{k}, y_{k}\right)\right\}_{k \in \mathbb{N}} \subset \Xi$ be a bounded sequence in $B V(\Omega) \times \mathbb{W}_{p}(\Omega)$. Then there is a pair $(u, y) \in B V(\Omega) \times \mathbb{W}_{p}(\Omega)$ such that, up to a subsequence, $\left(u_{k}, y_{k}\right) \stackrel{\tau}{\longrightarrow}(u, y)$ and $(u, y) \in \Xi$.

Proof. By Theorem 1 and compactness properties of the space $\mathbb{W}_{p}(\Omega)$, there exists a subsequence of $\left\{\left(u_{k}, y_{k}\right) \in \Xi\right\}_{k \in \mathbb{N}}$, still denoted by the same indices, and functions $u \in B V(\Omega)$ and $y \in \mathbb{W}_{p}(\Omega)$ such that

$$
u_{k} \rightarrow u \text { in } L^{1}(\Omega), y_{k} \rightarrow y \text { in } W_{0}^{2, p}\left(\Omega ; \Gamma_{D}\right) \text {, and, therefore, } y_{k} \rightarrow y \text { in } W_{0}^{1, p}(\Omega) .
$$

Then by Lemma 1, we have

$$
\lim _{k \rightarrow \infty} \int_{\Omega} u_{k} \Delta \varphi \Delta y_{k} \mathrm{~d} x=\int_{\Omega} u \Delta \varphi \Delta y \mathrm{~d} x, \quad \forall \varphi \in C_{0}^{\infty}\left(\Omega ; \Gamma_{D}\right) .
$$

It remains to show that the limit pair $(u, y)$ is related by inequality (21) and satisfies the state constraints (31). With that in mind we write down the Minty relation for $\left(u_{k}, y_{k}\right)$ :

$$
\begin{aligned}
& \int_{\Omega} u_{k}|\Delta \varphi|^{p-2} \Delta \varphi\left(\Delta \varphi-\Delta y_{k}\right) \mathrm{d} x+\int_{\Omega} F(x, \varphi)\left(\varphi-y_{k}\right) \mathrm{d} x \\
& \geq\left\langle f, \varphi-y_{k}\right\rangle_{W^{-2, q}\left(\Omega ; \Gamma_{D}\right) ; W_{0}^{2, p}\left(\Omega ; \Gamma_{D}\right)}, \quad \forall \varphi \in C_{0}^{\infty}\left(\Omega ; \Gamma_{D}\right) .
\end{aligned}
$$

In view of (34) and Lemma 1, we have

$$
\begin{aligned}
& \lim _{k \rightarrow \infty} \int_{\Omega}|\Delta \varphi|^{p} u_{k} \mathrm{~d} x=\int_{\Omega}|\Delta \varphi|^{p} u \mathrm{~d} x, \\
& \lim _{k \rightarrow \infty} \int_{\Omega} u_{k}|\Delta \varphi|^{p-2} \Delta \varphi \Delta y_{k} \mathrm{~d} x=\int_{\Omega} u|\Delta \varphi|^{p-2} \Delta \varphi \Delta y \mathrm{~d} x .
\end{aligned}
$$

Moreover, due to the compactness of the Sobolev imbedding $W_{0}^{2, p}\left(\Omega ; \Gamma_{D}\right) \hookrightarrow L^{r}(\Omega)$ for $r<p^{*}$, we have

$$
\int_{\Omega} F(x, \varphi)\left(\varphi-y_{k}\right) \mathrm{d} x=\int_{\Omega} F(x, \varphi)(\varphi-y) \mathrm{d} x+J_{k},
$$

where Hölder's inequality yields

$$
\left|J_{k}\right|:=\left|\int_{\Omega} F(x, \varphi)\left(y-y_{k}\right) \mathrm{d} x\right|^{\text {by }(12)} \leq\left(C_{1} \int_{\Omega}|\varphi|^{r} \mathrm{~d} x\right)^{\frac{r-1}{r}}\left\|y-y_{k}\right\|_{L^{r}(\Omega)} \rightarrow 0 \text { as } k \rightarrow \infty .
$$

We, thus, can pass to the limit in relation (35) as $k \rightarrow \infty$ and arrive at the inequality (21), which means that $y \in \mathbb{W}_{p}(\Omega)$ is a weak solution to the boundary value problem (15)-(16). Since the injections (6) are compact and the cone $L_{+}^{p}\left(\Gamma_{S}\right)$ is closed with respect to the strong convergence in $L^{p}\left(\Gamma_{S}\right)$, it follows that $\frac{\partial y_{k}}{\partial v} \rightarrow \frac{\partial y}{\partial v}$ 
strongly in $L^{p}\left(\Gamma_{S}\right)$ and, hence,

$$
\lim _{k \rightarrow \infty} \gamma_{1}\left(y_{k}\right)=\gamma_{1}(y) \in L_{+}^{p}\left(\Gamma_{S}\right) \text { and } \gamma_{1}(y) \in \zeta^{\max }-L_{+}^{p}\left(\Gamma_{S}\right) .
$$

This fact together with $u \in \mathfrak{A}_{a d}$ leads us to the conclusion: $(u, y) \in \Xi$, i.e. the limit pair $(u, y)$ is feasible to optimal control problem (19). The proof is complete.

In conclusion of this section, we give the existence result for optimal pairs to problem (19).

Theorem 4. Assume that, for given distributions $f \in W^{-2, q}\left(\Omega ; \Gamma_{D}\right), y_{d} \in L^{2}(\Omega)$, and $\zeta^{\max } \in L^{p}(\partial \Omega)$, the Hypothesis $\left(H_{1}\right)$ is valid. Then optimal control problem (19) admits at least one solution $\left(u^{o p t}, y^{o p t}\right) \in B V(\Omega) \times \mathbb{W}_{p}(\Omega)$.

Proof. Since the set $\Xi$ is nonempty and the cost functional is bounded from below on $\Xi$, it follows that there exists a minimizing sequence $\left\{\left(u_{k}, y_{k}\right) \in \Xi\right\}_{k \in \mathbb{N}}$ to problem (19). Then the inequality

$$
\inf _{(u, y) \in \Xi} I(u, y)=\lim _{k \rightarrow \infty}\left[\int_{\Omega}\left|y_{k}(x)-y_{d}(x)\right|^{2} \mathrm{~d} x+\int_{\Omega}\left|D u_{k}\right|\right]<+\infty,
$$

implies the existence of a constant $C>0$ such that

$$
\sup _{k \in \mathbb{N}} \int_{\Omega}\left|D u_{k}\right| \leq C .
$$

Hence, in view of the definition of the class of admissible controls $\mathfrak{A}_{\text {ad }}$ and a priori estimate (24), the sequence $\left\{\left(u_{k}, y_{k}\right) \in \Xi\right\}_{k \in \mathbb{N}}$ is bounded in $B V(\Omega) \times \mathbb{W}_{p}(\Omega)$. Therefore, by Theorem 3 , there exist functions $u^{*} \in \mathfrak{A}_{a d}$ and $y^{*} \in \mathbb{W}_{p}(\Omega)$ such that $\left(u^{*}, y^{*}\right) \in \Xi$ and, up to a subsequence, $u_{k} \rightarrow u^{*}$ weakly-* in $B V(\Omega)$ and $y_{k} \rightarrow y^{*}$ weakly in $W_{0}^{2, p}\left(\Omega ; \Gamma_{D}\right)$. To conclude the proof, it is enough to show that the cost functional $I$ is lower semicontinuous with respect to the $\tau$-convergence. Since $y_{k} \rightarrow y^{*}$ strongly in $L^{p}(\Omega)$ by Sobolev embedding theorem, it follows that

$$
\begin{aligned}
& \lim _{k \rightarrow \infty} \int_{\Omega}\left|y_{k}(x)-y_{d}(x)\right|^{2} \mathrm{~d} x=\int_{\Omega}\left|y^{*}(x)-y_{d}(x)\right|^{2} \mathrm{~d} x \text { and, } \\
& \liminf _{k \rightarrow \infty} \int_{\Omega}\left|D u_{k}\right| \geq \int_{\Omega}\left|D u^{*}\right| \text { by (10). }
\end{aligned}
$$

Thus,

$$
I\left(u^{*}, y^{*}\right) \leq \liminf _{k \rightarrow \infty} I\left(u_{k}, y_{k}\right)=\inf _{(u, y) \in \Xi} I(u, y) .
$$

Hence, $\left(u^{*}, y^{*}\right)$ is an optimal pair, and we arrive at the required conclusion.

\section{Henig Relaxation of State-Constrainted OCP (19)}

The main goal of this section is to provide a regularization of the pointwise state constraints by replacing the ordering cone $\Lambda:=L_{+}^{p}\left(\Gamma_{S}\right)$ (see (32)) by its solid Henig approximation $(\Lambda)_{\varepsilon}$ (see [21]-[24]) and show that the conical regularization approach leads to a family of optimization problems such that their solutions can be obtained by solving the corresponding optimality system and the regularized solution $\tau$-converge in the limit as $\varepsilon \rightarrow 0$ to a solution of the original problem.

We begin with some formal descriptions and abstract results. Let $Z$ be a real normed space, and let $\Lambda \subset Z$ be a closed ordering cone in $Z$.

Definition 5.1. A nonempty convex subset $B$ of a nontrivial ordering cone $\Lambda \subset Z$ (i.e. $\Lambda \neq\left\{0_{Z}\right\}$, where $0_{z}$ is the zero element in $Z$ ) is called base of $\Lambda$ if for each element $z \in \Lambda \backslash\left\{0_{z}\right\}$ there is a unique representation $z=\mu b$ where $\mu>0$ and $b \in B$.

In what follows, we always assume that the ordering cone $\Lambda$ has a closed base $B \subset \Lambda$. We note that, in general, bases are not unique. We denote the norm of $Z$ by $\|\cdot\|_{Z}$, and for arbitrary elements $z_{1}, z_{2} \in Z$ we define

$$
z_{1} \leq_{\Lambda} z_{2} \Leftrightarrow z_{2}-z_{1} \in \Lambda \text { as well as } z_{1}<_{\Lambda} z_{2} \Leftrightarrow{ }_{2}-z_{1} \in \Lambda \backslash\left\{0_{z}\right\} .
$$

In order to introduce a representation for a base of $\Lambda$, let $Z^{*}$ be the topological dual space of $Z$, and let 
$\langle\cdot, \cdot\rangle_{Z^{*}, Z}$ be the dual pairing. Moreover, by

$$
\Lambda^{*}:=\left\{z^{*} \in Z^{*} \mid\left\langle z^{*}, z\right\rangle_{z^{*}, Z} \geq 0 \forall z \in \Lambda\right\}
$$

and

$$
\Lambda^{\#}:=\left\{z^{*} \in Z^{*} \mid\left\langle z^{*}, z\right\rangle_{z^{*}, z}>0 \forall z \in \Lambda \backslash\left\{0_{z}\right\}\right\}
$$

we define the dual cone and the quasi-interior of the dual cone of $\Lambda$, respectively. Using the definition of the dual cone, the ordering cone $\Lambda$ can be characterized as follows (see [25], Lemma 3.21):

$$
\Lambda=\left\{z \in Z \mid\left\langle z^{*}, z\right\rangle_{z^{*}, Z} \geq 0 \forall z^{*} \in \Lambda^{*}\right\}
$$

Due to Lemma 1.28 in [25], we can give the following result.

Lemma 2. Let $\Lambda \subset Z$ be a nontrivial ordering cone in a Banach space $Z$. Then the set $B:=\left\{z \in \Lambda \mid\left\langle z^{*}, z\right\rangle_{Z^{*}, Z}=1\right\}$ is a base of $\Lambda$ for every $z^{*} \in \Lambda^{\#}$. Moreover, if $\Lambda$ is reproducing in $Z$, i.e. if $\Lambda-\Lambda=Z$, and if $B$ is a base of $\Lambda$, then there is an element $z^{*} \in \Lambda^{\#}$ satisfying $B:=\left\{z \in \Lambda \mid\left\langle z^{*}, z\right\rangle_{z^{*}, Z}=1\right\}$.

Remark 5.3. As follows from Lemma 2, the set

$$
B:=\left\{\xi \in L_{+}^{p}\left(\Gamma_{S}\right) \mid \int_{\Gamma_{S}} \xi \mathrm{d} \mathcal{H}^{N-1}=1\right\}
$$

is a closed base of ordering cone $\Lambda:=L_{+}^{p}\left(\Gamma_{S}\right)$.

Now, we are prepared to introduce the definition of a so-called Henig dilating cone (see Zhuang, [24]) which is based on the existence of a closed base of ordering cone $\Lambda$.

Definition 5.2. Let $Z$ be a normed space, and let $\Lambda \subset Z$ be a closed ordering cone with a closed base $B$. Choosing $\varepsilon>0$ arbitrarily, the corresponding Henig dilating cone is defined by

$$
\Lambda_{\varepsilon}(B):=\mathrm{cl}_{\|\cdot\|_{Z}}\left(\operatorname{cone}\left(B+B_{\varepsilon}\left(0_{Z}\right)\right)\right):=\mathrm{cl}_{\|\cdot\|_{z}}\left(\left\{\mu z \mid \mu \geq 0, z \in B+B_{\varepsilon}\left(0_{Z}\right)\right\}\right),
$$

where $\frac{1}{\varepsilon} B_{\varepsilon}\left(0_{Z}\right):=\left\{v \in Z \mid\|v\|_{Z} \leq 1\right\}$ is the closed unit ball in $Z$ centered at the origin.

It is clear that $\Lambda_{\varepsilon}(B)$ depends on the particular choice of $B$. As follows from this definition, $\operatorname{int}(\Lambda)_{\varepsilon}(B) \neq \varnothing$ for every $\varepsilon>0$, i.e. Henig dilating cone is proper solid. Moreover, we have the following properties of such cones (see [24] [26]).

Proposition 5. Let $Z$ be a normed space, and let $\Lambda \subset Z$ be a closed ordering cone with a closed base $B$. Choosing $\varepsilon \in(0, \delta)$, where

$$
\delta:=\inf \left\{\|b\|_{Z} \mid b \in B\right\}>0
$$

the following statements hold true.

1) $\Lambda_{\varepsilon}(B)$ is pointed, i.e. $\Lambda_{\varepsilon}(B) \cap\left(-\Lambda_{\varepsilon}(B)\right)=\left\{0_{Z}\right\}$;

2) $\Lambda_{\varepsilon}(B) \subset \Lambda_{\varepsilon+\gamma}(B) \forall \gamma>0$;

3) $\Lambda_{\varepsilon}(B)=$ cone $\left(\mathrm{cl}_{\|\cdot\|_{Z}}\left(B+B_{\varepsilon}\left(0_{Z}\right)\right)\right)$;

4) $\Lambda=\bigcap_{0<\varepsilon<\delta} \Lambda_{\varepsilon}(B)$;

5) the implication

$$
\begin{aligned}
& \xi \in(\Lambda)_{\varepsilon}(B) \Rightarrow \frac{\varepsilon}{\kappa+\varepsilon}\|\xi\|_{Z}+\xi \notin(-\Lambda), \\
& \text { i.e. } \xi \nless_{\Lambda}-\frac{\varepsilon}{\kappa+\varepsilon}\|\xi\|_{Z}
\end{aligned}
$$

holds true with $\kappa=\sup \left\{\|\xi\|_{Z}: \zeta \in B\right\}$. 
In the context of constraint qualifications problem, the following result plays an important role.

Proposition 6. Let $Z$ be a normed space, and let $\Lambda \subset Z$ be a closed ordering cone with a closed base $B$. Choosing $\varepsilon \in(0, \delta)$ arbitrarily, where $\delta$ is defined by (37), the inclusion

$$
\Lambda \subset\left\{0_{z}\right\} \cup \operatorname{int}\left(\Lambda_{\varepsilon}(B)\right)
$$

holds true.

Proof. Let $z \in \Lambda \backslash\left\{0_{z}\right\}$ be chosen arbitrarily. By the definition of a base there is a unique representation $z=\lambda b$ with $\lambda>0$ and $b \in B$. Obviously,

$$
z \in \operatorname{int}\left(\{\lambda b\}+B_{\lambda \varepsilon}\left(0_{Z}\right)\right)=\operatorname{int}\left(B_{\lambda \varepsilon}(\lambda b)\right)
$$

holds true. Let's assume for a moment that

$$
B_{\lambda \varepsilon}(\lambda b) \subseteq \operatorname{cone}\left(\{b\}+B_{\varepsilon}\left(0_{Z}\right)\right) .
$$

Then we obtain

$$
z \in \operatorname{int}\left(\operatorname{cone}\left(\{b\}+B_{\varepsilon}\left(0_{z}\right)\right)\right) \subseteq \operatorname{int}\left(\operatorname{cone}\left(B+B_{\varepsilon}\left(0_{z}\right)\right)\right)=\operatorname{int}\left(\Lambda_{\varepsilon}(B)\right),
$$

which completes the proof. In order to show (40), let $x \in B_{\lambda \varepsilon}(\lambda b)$ be chosen arbitrarily, i.e.

$$
\|x-\lambda b\|_{Z} \leq \lambda \varepsilon
$$

Then

$$
\left\|\frac{x}{\lambda}-b\right\|_{Z}=\frac{1}{\lambda}\|x-\lambda b\|_{Z} \leq \frac{\lambda \varepsilon}{\lambda}=\varepsilon
$$

yields

$$
x \in\left\{\mu y \mid\|y-b\|_{z} \leq \varepsilon, \mu \geq 0\right\}=\operatorname{cone}\left(\{b\}+B_{\varepsilon}\left(0_{z}\right)\right) .
$$

As a result, (40) is satisfied.

Remark 5.2. The following property, coming from Proposition 6, turns out rather useful: in order to prove $z \in \operatorname{int}\left(\Lambda_{\varepsilon}(B)\right)$, it is sufficient to check whether $z \in \Lambda \backslash\left\{0_{z}\right\}$.

The following result shows that Henig dilating cones $\Lambda_{\varepsilon}(B)$ possess good approximation properties.

Proposition 7. Let $\Lambda$ be a closed ordering cone in a normed space $Z$, and let $B$ be an arbitrary closed base of $\Lambda$. Let parameter $\delta$ be defined as in (37), and let $\left(\varepsilon_{k}\right)_{k \in \mathbb{N}} \subset(0, \delta)$ be a monotonically decreasing sequence such that $\lim _{k \rightarrow \infty} \varepsilon_{k}=0$. Then the sequence of cones $\left\{\Lambda_{\varepsilon_{k}}(B)\right\}_{k \in \mathbb{N}}$ converges to $\Lambda$ in Kuratowski sense with respect to the norm topology of $Z$ as $k$ tends to infinity, that is

$$
K-\liminf _{k \rightarrow \infty} \Lambda_{\varepsilon_{k}}(B)=\Lambda=K-\limsup _{k \rightarrow \infty} \Lambda_{\varepsilon_{k}}(B),
$$

where

$$
\begin{aligned}
& K-\liminf _{k \rightarrow \infty} \Lambda_{\varepsilon_{k}}(B):=\{z \in Z \mid \text { for all neighborhoods } N \text { of } z \text { there is } a \\
& \left.k_{0} \in \mathbb{N} \text { such that } N \cap \Lambda_{\varepsilon_{k}}(B) \neq \varnothing \forall k \geq k_{0}\right\}, \\
& K-\limsup _{k \rightarrow \infty} \Lambda_{\varepsilon_{k}}(B):=\left\{z \in Z \mid \text { for all neighborhoods } N \text { of } z \text { and every } k_{0} \in \mathbb{N}\right. \\
& \text { there is } \left.a k \geq k_{0} \text { such that } N \cap \Lambda_{\varepsilon_{k}}(B) \neq \varnothing\right\} \text {. }
\end{aligned}
$$

Proof. Let $z \in \Lambda$ be chosen arbitrarily. Then $N \cap \Lambda \neq \varnothing$ holds true for every neighborhood $N$ of $z$, and due to the inclusions $\Lambda \subset \Lambda_{\varepsilon_{k}} \quad \forall k \in \mathbb{N}$, we see that $N \cap \Lambda_{\varepsilon_{k}} \neq \varnothing$ for all $k \in \mathbb{N}$. Hence,

$$
\Lambda \subseteq K-\liminf _{k \rightarrow \infty} \Lambda_{\varepsilon_{k}}(B) .
$$

Taking into account the inclusion (41) and the fact that 


$$
K-\liminf _{k \rightarrow \infty} \Lambda_{\varepsilon_{k}}(B) \subseteq K-\limsup _{k \rightarrow \infty} \Lambda_{\varepsilon_{k}}(B),
$$

we get

$$
\Lambda \subseteq K-\liminf _{k \rightarrow \infty} \Lambda_{\varepsilon_{k}}(B) \subseteq K-\limsup _{k \rightarrow \infty} \Lambda_{\varepsilon_{k}}(B) .
$$

To show that the sequence $\left\{\Lambda_{\varepsilon_{k}}(B)\right\}_{k \in \mathbb{N}}$ converges to $\Lambda$ in Kuratowski sense, it remains to show

$$
K-\limsup _{k \rightarrow \infty} \Lambda_{\varepsilon_{k}}(B) \subseteq \Lambda \text {. }
$$

However, the inclusion (43) is equivalent to

$$
(Z \backslash \Lambda) \subseteq\left(Z \backslash K-\limsup _{k \rightarrow \infty} \Lambda_{\varepsilon_{k}}(B)\right) .
$$

Let $\bar{Z} \in Z \backslash \Lambda$ be an arbitrarily element. Since $\Lambda$ is closed, there is an open neighborhood $\bar{N}$ of $\bar{Z}$ with respect to the norm topology of $Z$ such that $\bar{N} \cap \Lambda=\varnothing$. By Proposition 5 (see item (4)), there is a sufficiently large index $k_{0} \in \mathbb{N}$ such that

$$
\bar{N} \cap \Lambda_{\varepsilon_{k}}(B)=\varnothing \quad \forall k \geq k_{0} .
$$

This implies

$$
\bar{Z} \in Z \backslash \limsup _{k \rightarrow \infty} \Lambda_{\varepsilon_{k}}(B) .
$$

Combining (42), (43), and (44), we arrive at the relation

$$
\Lambda \subseteq K-\liminf _{k \rightarrow \infty} \Lambda_{\varepsilon_{k}}(B) \subseteq K-\limsup _{k \rightarrow \infty} \Lambda_{\varepsilon_{k}}(B) \subseteq \Lambda .
$$

Thus, $\Lambda=K-\lim _{k \rightarrow \infty} \Lambda_{\varepsilon_{k}}(B)$ and the proof is complete.

Taking these results into account, we associate with OCP (19) the following family of Henig relaxed problems

$$
\operatorname{Minimize}\left\{I(u, y)=\int_{\Omega}\left|y-y_{d}\right|^{2} \mathrm{~d} x+\int_{\Omega}|D u|\right\}
$$

subject to the constraints

$$
\begin{aligned}
& \Delta_{p}^{2}(u, y)+F(y)=f \text { in }\left(C_{0}^{\infty}\left(\Omega ; \Gamma_{D}\right)\right)^{*}, \\
& \gamma_{0}(y)=0 \text { in } W^{2-1 / p, p}(\partial \Omega), \\
& \gamma_{1}(y)=0 \text { in } W^{1 / q, p}\left(\Gamma_{D}\right), \\
& \gamma_{0}(\Delta y)=0 \text { in } W^{-1 / p, p}\left(\Gamma_{S}\right), \\
& \gamma_{1}(y) \in\left(L_{+}^{p}\left(\Gamma_{S}\right)\right)_{\varepsilon}(B), \\
& \zeta^{\max }-\gamma_{1}(y) \in\left(L_{+}^{p}\left(\Gamma_{S}\right)\right)_{\varepsilon}(B) \\
& u \in \mathfrak{A}_{a d},
\end{aligned}
$$

or in a more compact form each of these problems can be stated as follows

$$
\inf _{(u, y) \in \Xi_{\varepsilon}} I(u, y), \quad \forall \varepsilon \in(0, \delta),
$$

where

$$
\delta=\inf \left\{\|\xi\|_{L^{p}\left(\Gamma_{S}\right)}: \xi \in B\right\},
$$

the base $B$ takes the form (36), and the feasible set $\Xi_{\varepsilon} \subset B V(\Omega) \times \mathbb{W}_{p}(\Omega)$ we define as follows: $(u, y) \in \Xi_{\varepsilon}$ if and only if $u \in \mathfrak{A}_{a d}, I(u, y)<+\infty, y \in \mathbb{W}_{p}(\Omega)$, the pair $(u, y)$ is related by the Minty inequality (21), and 


$$
\gamma_{1}(y) \in\left(L_{+}^{p}\left(\Gamma_{S}\right)\right)_{\varepsilon}(B), \quad \zeta^{\max }-\gamma_{1}(y) \in\left(L_{+}^{p}\left(\Gamma_{S}\right)\right)_{\varepsilon}(B) .
$$

Here, $\left(L_{+}^{p}\left(\Gamma_{S}\right)\right)_{s}(B)$ stands for the corresponding Henig dilating cone.

Since, by Proposition 6, the inclusion $\Xi \subseteq \Xi_{\varepsilon}$ holds true for all $\varepsilon>0$, it is reasonable to call the OCP (47) a Henig relaxation of OCP (19). Moreover, as obviously follows from Proposition 7 , the convergence $\Xi_{\varepsilon} \stackrel{\varepsilon \rightarrow 0}{\longrightarrow} \Xi$ in Kuratowski sense holds true with respect to the $\tau$-topology on $B V(\Omega) \times \mathbb{W}_{p}(\Omega)$.

We are now in a position to show that using the relaxation approach we can reduce the main suppositions of Theorem 4. In particular, we can characterize Hypothesis $\left(H_{1}\right)$ by the non-emptiness properties of feasible sets $\Xi_{\varepsilon}$ for the corresponding Henig relaxed problems.

Theorem 8. Let $\left\{\varepsilon_{k}\right\}_{k \in \mathbb{N}} \subset(0, \delta)$ be a monotonically decreasing sequence converging to 0 as $k \rightarrow \infty$. Then, for given distributions $f \in W^{-2, q}\left(\Omega ; \Gamma_{D}\right), \quad y_{d} \in L^{2}(\Omega)$, and $\zeta^{\max } \in L^{p}(\partial \Omega)$, the Hypothesis $\left(H_{1}\right)$ implies that the Henig relaxed problem (47) has a nonempty set of feasible solutions $\Xi_{\varepsilon}$ for all $\varepsilon=\varepsilon_{k}, k \in \mathbb{N}$. And vice versa, if there exists a sequence $\left\{\left(u^{k}, y^{k}\right)\right\}_{k \in \mathbb{N}}$ satisfying conditions

$$
\left(u^{k}, y^{k}\right) \in \Xi_{\varepsilon_{k}} \text { for all } k \in \mathbb{N} \text {, and } \sup _{k \in \mathbb{N}} I\left(u^{k}, y^{k}\right)<+\infty \text {, }
$$

then the sequence $s\left\{\left(u^{k}, y^{k}\right)\right\}_{k \in \mathbb{N}}$ is $\tau$-compact and each of its $\tau$-cluster pairs is a feasible solution to the original OCP (19).

Proof. Since the implication $(\Xi \neq \varnothing) \Rightarrow\left(\Xi_{\varepsilon} \neq \varnothing\right.$ for all $\left.\varepsilon>0\right)$ is obvious by Proposition 7 , we concentrate on the proof of the inverse statement - property (50) implies the existence of at least one pair $(u, y)$ such that $(u, y) \in \Xi$.

Let $\left\{\left(u^{k}, y^{k}\right)\right\}_{k \in \mathbb{N}}$ be an arbitrary sequence with property: $\left(u^{k}, y^{k}\right) \in \Xi_{\varepsilon_{k}}$ for all $k \in \mathbb{N}$. Since the set $\mathfrak{A}_{a d}$ and a priory estimate (24) do not depend on parameter $\varepsilon_{k}$ and the condition (50) implies $\sup _{k \in \mathbb{N}} \int_{\Omega}\left|D u_{k}\right| \leq \infty$, it follows by compactness arguments (see the proof of Theorem 4) that there exist a subsequence of $\left\{\left(u^{k}, y^{k}\right)\right\}_{k \in \mathbb{N}}$ (still denoted by the same index) and a pair $\left(u^{*}, y^{*}\right) \in \mathfrak{A}_{a d} \times \mathbb{W}_{p}(\Omega)$ such that

$$
\left(u^{k}, y^{k}\right) \stackrel{\tau}{\longrightarrow}\left(u^{*}, y^{*}\right) \text { as } k \rightarrow \infty .
$$

Closely following the proof of Theorem 3 , it can be shown that the limit pair $\left(u^{*}, y^{*}\right)$ is such that $u^{*} \in \mathfrak{A}_{a d}$, $J\left(u^{*}, y^{*}\right)<+\infty$, and function $y^{*} \in \mathbb{W}_{p}(\Omega)$ is a weak solution to the boundary value problem (15) - (16). Moreover, in view of the compactness properties of injections (6), we may suppose that

$$
\gamma_{1}\left(y^{k}\right) \rightarrow \gamma_{1}(y) \text { strongly in } L^{p}\left(\Gamma_{S}\right) \text { as } k \rightarrow \infty .
$$

It remains to establish the inclusions

$$
\gamma_{1}\left(y^{*}\right) \in L_{+}^{p}\left(\Gamma_{S}\right), \quad \zeta^{\max }-\gamma_{1}\left(y^{*}\right) \in L_{+}^{p}\left(\Gamma_{S}\right),
$$

By contraposition, let us assume that $\xi^{*}:=\zeta^{\max }-\gamma_{1}\left(y^{*}\right) \in L^{p}\left(\Gamma_{S}\right) \backslash L_{+}^{p}\left(\Gamma_{S}\right)$. Since the cone $L_{+}^{p}\left(\Gamma_{S}\right)$ is closed, it follows that there is a neighborhood $\mathcal{N}\left(\xi^{*}\right)$ of $\xi^{*}$ in $L^{p}\left(\Gamma_{S}\right)$ such that $\mathcal{N}\left(\xi^{*}\right) \cap L_{+}^{p}\left(\Gamma_{S}\right)=\varnothing$. Using the fact that

$$
L_{+}^{p}\left(\Gamma_{S}\right) \subset\left(L_{+}^{p}\left(\Gamma_{S}\right)\right)_{\varepsilon_{k}}(B) \subseteq\left(L_{+}^{p}\left(\Gamma_{S}\right)\right)_{\varepsilon_{l}}(B), \quad \forall k \geq l,
$$

by Proposition 7 and definition of the Kuratowski limit, it is easy to conclude the existence of an index $k_{0} \in \mathbb{N}$ such that

$$
\mathcal{N}\left(\xi^{*}\right) \cap\left(L_{+}^{p}\left(\Gamma_{S}\right)\right)_{\varepsilon_{k}}(B)=\varnothing, \quad \forall k \geq k_{0} .
$$

However, in view of the strong convergence property (51), there is an index $k_{1} \in \mathbb{N}$ satisfying

$$
\xi^{k} \in \mathcal{N}\left(\xi^{*}\right), \quad \forall k \geq k_{1}
$$

Combining (53) and (54), we finally obtain

$$
\xi^{k}=\zeta^{\max }-\gamma_{1}\left(y^{k}\right) \in L^{p}\left(\Gamma_{S}\right) \backslash\left(L_{+}^{p}\left(\Gamma_{S}\right)\right)_{\varepsilon_{k}}(B), \quad \forall k \geq \max \left\{k_{0}, k_{1}\right\} .
$$


This, however, is a contradiction to

$$
\zeta^{\max }-\gamma_{1}\left(y^{k}\right) \in\left(L_{+}^{p}\left(\Gamma_{S}\right)\right)_{\varepsilon_{k}}(B), \quad \forall k \in \mathbb{N} .
$$

Thus, $\zeta^{\max }-\gamma_{1}\left(y^{*}\right) \in L_{+}^{p}\left(\Gamma_{S}\right)$. In the same manner it can be shown that $\gamma_{1}\left(y^{*}\right) \in L_{+}^{p}\left(\Gamma_{s}\right)$. Hence, the pair $\left(u^{*}, y^{*}\right)$ is feasible for OCP (19).

As an obvious consequence of this Theorem and Theorem 4, we have the following noteworthy property of the Henig relaxed problems (47).

Corollary 2. Let $f \in W^{-2, q}\left(\Omega ; \Gamma_{D}\right), \quad y_{d} \in L^{2}(\Omega)$, and $\zeta^{\max } \in L^{p}(\partial \Omega)$ be given distribution. Then the Henig relaxed problem (47) is solvable for each $\varepsilon \in(0, \delta)$ provided Hypothesis $\left(H_{1}\right)$ is satisfied.

The next result is crucial in this section. We show that some optimal solutions for the original OCP (19) can be attained by solving the corresponding Henig relaxed problems (45)-(46). However, we do not claim that the entire set of the solutions to OCP (19) can be restored in such way.

Theorem 9. Let $f \in W^{-2, q}\left(\Omega ; \Gamma_{D}\right), \quad y_{d} \in L^{2}(\Omega)$, and $\zeta^{\max } \in L^{p}(\partial \Omega)$ be given distributions. Let $\left\{\varepsilon_{k}\right\}_{k \in \mathbb{N}} \subset(0, \delta)$ be a monotonically decreasing sequence such that $\varepsilon_{k} \rightarrow 0$ as $k \rightarrow \infty$, where $\delta>0$ is defined by (48). Let $\left\{\left(u^{k, 0}, y^{k, 0}\right) \in \Xi_{\varepsilon_{k}}\right\}_{k \in \mathbb{N}}$ be a sequence of optimal solutions to the Henig relaxed problems (45)(46) such that

$$
\sup _{k \in \mathbb{N}}\left\|u^{k, 0}\right\|_{B V(\Omega)}<+\infty .
$$

Then there is a subsequence $\left\{\left(u^{k_{i}, 0}, y^{k_{i}, 0}\right)\right\}_{i \in \mathbb{N}}$ of $\left\{\left(u^{k, 0}, y^{k, 0}\right)\right\}_{k \in \mathbb{N}}$ and a pair $\left(u^{0}, y^{0}\right)$ such that

$$
\begin{aligned}
& \left(u^{k_{i}, 0}, y^{k_{i}, 0}\right) \stackrel{\tau}{\longrightarrow}\left(u^{0}, y^{0}\right) \text { as } i \rightarrow \infty, \\
& \left(u^{0}, y^{0}\right) \in \Xi, \text { and } I\left(u^{0}, y^{0}\right)=\inf _{(u, y) \in \Xi} I(u, y) .
\end{aligned}
$$

Proof. In view of a priory estimate (24), the uniform boundedness of optimal controls with respect to $B V$-norm (55) implies the fulfilment of condition (50) $)_{2}$. Hence, the compactness property (56) and the inclusion $\left(u^{0}, y^{0}\right) \in \Xi$ are a direct consequence of Theorem 8. It remains to show that the limit pair $\left(u^{0}, y^{0}\right)$ is a solution to OCP (19). Indeed, the condition $\left(u^{0}, y^{0}\right) \in \Xi$ implies the fulfilment of Hypothesis $\left(H_{1}\right)$. Hence, by Theorem 4, the original OCP (19) has a nonempty set of solutions. Let $\left(u^{*}, y^{*}\right)$ be one of them. Then the following inequality is obvious

$$
I\left(u^{*}, y^{*}\right) \leq I\left(u^{0}, y^{0}\right) .
$$

On the other hand, by Proposition 5 (see property (4)), we have $\left(u^{*}, y^{*}\right) \in \Xi_{\varepsilon_{k_{i}}}$ for every $i \in \mathbb{N}$. Since $\left\{\left(u^{k_{i}, 0}, y^{k_{i}, 0}\right)\right\}_{i \in \mathbb{N}}$ are the solutions to the corresponding relaxed problems (47), it follows that

$$
\inf _{(u, y) \in \Xi_{\varepsilon_{k_{i}}}} I(u, y)=I\left(u^{k_{i}, 0}, y^{k_{i}, 0}\right) \leq I\left(u^{*}, y^{*}\right), \quad \forall i \in \mathbb{N} .
$$

As a result, taking into account the relations (58) and (59), and the lower semicontinuity property of the cost functional $I$ with respect to the $\tau$-convergence, we finally get

$$
\begin{aligned}
\inf _{(u, y) \in \Xi} I(u, y) & =I\left(u^{*}, y^{*}\right) \stackrel{\text { by (59) }}{\geq} \liminf _{i \rightarrow \infty} I\left(u^{k_{i}, 0}, y^{k_{i}, 0}\right) \\
& \geq \liminf _{i \rightarrow \infty} I\left(u^{k_{i}, 0}, y^{k_{i}, 0}\right) \geq I\left(u^{0}, y^{0}\right) \stackrel{\text { by }(58)}{\geq} I\left(u^{*}, y^{*}\right) .
\end{aligned}
$$

Thus,

$$
\inf _{(u, y) \in \Xi} I(u, y)=\lim _{i \rightarrow \infty} I\left(u^{k_{i}, 0}, y^{k_{i}, 0}\right)=I\left(u^{0}, y^{0}\right),
$$

and we arrive at the desired property $(57)_{2}$. The proof is complete.

Remark 5.3. It is worth to note that condition (55) can be omitted if the original OCP (19) is regular, that is when Hypothesis $\left(H_{1}\right)$ is valid. Indeed, let us assume that $\Xi \neq \varnothing$ and $(\hat{u}, \hat{y}) \in \Xi$ is an arbitrary pair. Then $(\hat{u}, \hat{y})$ is feasible to each Henig relaxed problems (45)-(46), and, hence, 


$$
\inf _{(u, y) \in \Xi_{\varepsilon_{k}}} I(u, y)=I\left(u^{k, 0}, y^{k, 0}\right) \leq I(\hat{u}, \hat{y}), \quad \forall k \in \mathbb{N} .
$$

Since, by Proposition 6, the inclusion $\Xi \subseteq \Xi_{\varepsilon_{k}}$ holds true for all $\varepsilon_{k}>0$, and the sequence $\left\{\Xi_{\varepsilon_{k}}\right\}_{k \in \mathbb{N}}$ is monotone in the following sense (because of the property (2) of Proposition 5)

$$
\Xi_{\varepsilon_{1}} \supseteq \Xi_{\varepsilon_{2}} \supseteq \ldots \supseteq \Xi_{\varepsilon_{k}} \supseteq \ldots \supseteq \Xi \neq \varnothing,
$$

it follows that

$$
\inf _{(u, y) \in \Xi_{\varepsilon_{1}}} I(u, y) \leq \cdots \leq \inf _{(u, y) \in \Xi_{\varepsilon_{k}}} I(u, y) \leq \cdots \leq \inf _{(u, y) \in \Xi} I(u, y) \leq I(\hat{u}, \hat{y}) .
$$

As a result, (60) leads to the estimate

$$
\sup _{k \in \mathbb{N}}\left\|u^{k, 0}\right\|_{B V(\Omega)} \leq \sup _{k \in \mathbb{N}}\left[\int_{\Omega}\left|u^{k, 0}\right| \mathrm{d} x+\inf _{(u, y) \in \Xi_{\varepsilon_{k}}} I(u, y)\right] \leq\left\|\xi_{2}\right\|_{L^{\infty}(\Omega)}|\Omega|+I(\hat{u}, \hat{y})<+\infty .
$$

As was mentioned at the beginning of this section, the main benefit of the relaxed optimal control problems (45)-(46) comes from the fact that the Henig dilating cone $\left(L_{+}^{p}\left(\Gamma_{S}\right)\right)(B)$ has a nonempty topological interior. Hence, it gives a possibility to apply the Slater condition or the Robinson condition in order to characterize the optimal solutions for the state constrained OCP (19). On the other hand, this approach provides nice convergence properties for the solutions of relaxed problems (45)-(46). However, as follows from Theorems 8 and 9 (see also Remark 5.5), the most restrictive assumption deals with the regularity of the relaxed problems (45)-(46) for all $\varepsilon \in(0, \delta)$. So, if we reject the Hypothesis $\left(H_{1}\right)$, it becomes unclear, in general, whether the relaxed sets of feasible solutions $\Xi_{\varepsilon}$ are nonempty for all $\varepsilon \searrow 0$. In this case it makes sense to provide further relaxation for each of Henig problems (45)-(46). In particular, using the methods of variational inequalities, we show in the next section that original OCP (19) may admit the existence of the so-called weakened approximate solution which can be interpreted as an optimal solution to some optimization problem of a special form.

\section{Variational Inequality Approach to Regularization of OCP (19)}

As follows from Theorem 4, the existence of optimal solutions to the problem (19) can be obtained by using compactness arguments and the Hypothesis $\left(H_{1}\right)$. However, because of the state constraints (17) the fulfilment of Hypothesis $\left(H_{1}\right)$ is an open question even for the simplest situation. Nevertheless, in many applications it is an important task to find a feasible (or at least an approximately admissible, in a sense to be made precise) solution when both control and state constraints for the OCP are given. Thus, if the set of feasible solutions is rather "thin", it is reasonable to weaken the requirements on feasible solutions to the original OCP. In particular, it would be reasonable to assume that we may satisfy the state equation

$$
\langle A(u, y), \varphi\rangle_{\left(\mathbb{W}_{p}(\Omega)\right)^{*} ; \mathbb{W}_{p}(\Omega)}=\langle f, \varphi\rangle_{\left(\mathbb{W}_{p}(\Omega)\right)^{*} ; \mathbb{W}_{p}(\Omega)}, \forall \varphi \in \mathbb{W}_{p}(\Omega)
$$

and the corresponding state constraint

$$
y \in K:=\left\{v \in \mathbb{W}_{p}(\Omega) \mid \gamma_{1}(v) \in L_{+}^{p}\left(\Gamma_{S}\right), \zeta^{\max }-\gamma_{1}(v) \in L_{+}^{p}\left(\Gamma_{S}\right)\right\}
$$

with some accuracy. Here, the operator $A(\cdot, \cdot): B V(\Omega) \times \mathbb{W}_{p}(\Omega) \rightarrow\left(\mathbb{W}_{p}(\Omega)\right)^{*}$ is defined by the left-hand side of relation (29). For this purpose, we make use of the following observation: If a pair $(u, y)$ is feasible to the original problem, i.e. $(u, y) \in \Xi$, then this pair satisfies the relation

$$
\langle A(u, y), \zeta-y\rangle_{\left(\mathbb{W}_{p}(\Omega)\right)^{*} ; \mathbb{W}_{p}(\Omega)} \geq\langle f, \zeta-y\rangle_{\left(\mathbb{W}_{p}(\Omega)\right)^{*} ; \mathbb{W}_{p}(\Omega)}, \quad \forall \zeta \in K_{\varepsilon}
$$

for each $\varepsilon>0$, where $K_{\varepsilon}$ is defined as follows

$$
K_{\varepsilon}:=\left\{v \in \mathbb{W}_{p}(\Omega) \mid \gamma_{1}(v) \in\left(L_{+}^{p}\left(\Gamma_{S}\right)\right)_{\varepsilon}(B), \zeta^{\max }-\gamma_{1}(v) \in\left(L_{+}^{p}\left(\Gamma_{S}\right)\right)_{\varepsilon}(B)\right\} .
$$

Here, $\left(L_{+}^{p}\left(\Gamma_{S}\right)\right)_{\varepsilon}(B)$ is the corresponding Henig dilating cone.

Note that the reverse statement is not true in general. In fact, we discuss a variant of the penalization approach, called the "variational inequality (VI) method". This idea was first studied in [27]. Thus, if a pair $(u, y) \in \mathfrak{A}_{a d} \times K$ is related by variational inequality (61), then it is not necessary to suppose that $(u, y)$ satisfy the operator 
equation $A(u, y)=f$. In view of this, we can use the penalized term $\|A(u, y)-f\|_{\left(\mathbb{W}_{p}(\Omega)\right)^{*}}$ as a deviation measure in an associated cost functional. As a result, we arrive at the following penalized OCP:

$$
\operatorname{Minimize}\left\{\hat{I}_{\varepsilon}(u, y)=\int_{\Omega}\left|y-y_{d}\right|^{2} \mathrm{~d} x+\int_{\Omega}|D u|+\frac{1}{\varepsilon}\|A(u, y)-f\|_{\left(\mathbb{W}_{p}(\Omega)\right)^{*}}\right\}
$$

subject to the constraints

$$
\left.\begin{array}{l}
u \in \mathfrak{A}_{a d}, \quad y \in K_{\varepsilon}, \\
\langle A(u, y), \zeta-y\rangle_{\left(\mathbb{W}_{p}(\Omega)\right)^{*} ; \mathbb{W}_{p}(\Omega)} \geq\langle f, \zeta-y\rangle_{\left(\mathbb{W}_{p}(\Omega)\right)^{*} ; \mathbb{W}_{p}(\Omega)}, \forall \zeta \in K_{\varepsilon},
\end{array}\right\}
$$

or in a more compact form this problem can be stated as follows

$$
\inf _{(u, y) \in \hat{\Xi}_{\varepsilon}} \hat{I}_{\varepsilon}(u, y), \quad \forall \varepsilon \in(0, \delta)
$$

where $\delta>0$ is given by (48), the set $K_{\varepsilon}$ is defined in (62), and the set of feasible solutions $\hat{\Xi}_{\varepsilon} \subset B V(\Omega) \times \mathbb{W}_{p}(\Omega)$ we describe as follows:

$$
\hat{\Xi}_{\varepsilon}:=\left\{(u, y): u \in \mathfrak{A}_{a d}, \in K_{\varepsilon}, \hat{I}_{\varepsilon}(u, y)<+\infty \text {, and }(u, y) \text { satisfies VI (61) }\right\} .
$$

In this section we show that penalized OCP (65) is solvable for each $\varepsilon>0$ without any assumption about fulfilment of Hypothesis $\left(H_{1}\right)$. We also study the asymptotic properties of sequences of optimal pairs $\left\{\left(u_{\varepsilon}^{0}, y_{\varepsilon}^{0}\right)\right\}$. to problem (65) when the small parameter $\varepsilon>0$ varies in a strictly decreasing sequence of positive numbers converging to zero. We begin with the following result.

Lemma 3. Under assumptions (11)-(14), for every fixed $u \in U_{\partial}$ and $\varepsilon \in(0, \delta)$, the variational inequality (61) admits at least one solution $y=y(u)$ such that $y \in K_{\varepsilon}$.

Proof. Let $\varepsilon \in(0, \delta)$ be a fixed value. As follows from definition of the set $K_{\varepsilon}$ (see (62) and Remark 5.1), $K_{\varepsilon}$ is a nonempty convex closed subset of $\mathbb{W}_{p}(\Omega)$ with respect to the $\|\cdot\|_{p, \Delta}$-norm topology. Due to the assumptions (11)-(14), we have the following estimates

$$
\begin{aligned}
& \sup _{\|y\|_{p, \Delta}^{\leq \rho}}\|A(u, y)\|_{\left(\mathbb{W}_{p}(\Omega)\right)^{*}}=\sup _{\|y\|_{p, \Delta} \leq \rho\|\| \|_{p, \Delta} \leq 1}\langle A(u, y), v\rangle_{\left(\mathbb{W}_{p}(\Omega)\right)^{*} ; \mathbb{W}_{p}(\Omega)} \\
& =\sup _{\|y\|_{p, \Delta} \leq \rho} \sup _{\|v\|_{p, \Delta} \leq 1}\left[\int_{\Omega} u|\Delta y|^{p-2} \Delta y \Delta v \mathrm{~d} x+\int_{\Omega} F(x, y) v \mathrm{~d} x\right] \\
& \leq \sup _{\|y\|_{p, \Delta} \leq \rho\|\| \|_{p, \Delta} \leq 1} \sup _{\Omega}\left[\left\|\xi_{2}\right\|_{L^{\infty}(\Omega)}\|y\|_{p, \Delta}^{p-1}\|v\|_{p, \Delta}+C_{1}\|y\|_{L^{r}(\Omega)}^{r-1}\|v\|_{L^{r}(\Omega)}\right] \\
& \leq \sup _{\|y\|_{p, \Delta} \leq \rho\|\| \|_{p, \Delta} \leq 1} \sup _{\|}\left[\left\|\xi_{2}\right\|_{L^{\infty}(\Omega)}\|y\|_{p, \Delta}^{p-1}\|v\|_{p, \Delta}+C_{1} N_{*}^{r}\|y\|_{p, \Delta}^{r-1}\|v\|_{p, \Delta}\right] \\
& \leq\left\|\xi_{2}\right\|_{L^{\infty}(\Omega)} \rho^{p-1}+C_{1} N_{*}^{r} \rho^{r-1}<+\infty, \\
& \langle A(u, y), y\rangle_{\left(\mathbb{W}_{p}(\Omega)\right)^{*} ; \mathbb{W}_{p}(\Omega)} \geq \int_{\Omega} u|\Delta y|^{p} \mathrm{~d} x \geq \alpha\|y\|_{p, \Delta}^{p},
\end{aligned}
$$

where $N_{*}$ is the norm of the embedding operator $W^{2, p}(\Omega) \hookrightarrow L^{r}(\Omega)$. Hence, for every fixed $u \in \mathfrak{A}_{a d}$, the operator $A(u, \cdot): \mathbb{W}_{p}(\Omega) \rightarrow\left(\mathbb{W}_{p}(\Omega)\right)^{*}$ is bounded and coercive. Moreover, it is shown in [16, Proposition 2.42], the properties (11)-(14) ensure the following implication

$$
\begin{aligned}
& y_{k} \rightarrow y \text { in } W_{0}^{2, p}\left(\Omega ; \Gamma_{D}\right), \\
& \left.\left.\underset{k \rightarrow \infty}{\limsup _{k}\left\langle A\left(u, y_{k}\right), y_{k}-y\right\rangle_{\left(\mathbb{W}_{p}(\Omega)\right)^{*} ; \mathbb{W}_{p}(\Omega)} \leq 0}\right\}\right\} \\
& \Rightarrow\left\{\begin{array}{l}
\forall v \in W_{0}^{2, p}\left(\Omega ; \Gamma_{D}\right) \\
\langle A(u, y), y-v\rangle_{\left(\mathbb{W}_{p}(\Omega)\right)^{*} ; \mathbb{W}_{p}(\Omega)} \leq \liminf _{k \rightarrow \infty}\left\langle A\left(u, y_{k}\right), y_{k}-v\right\rangle_{\left(\mathbb{W}_{p}(\Omega)\right)^{*} ; \mathbb{W}_{p}(\Omega)} .
\end{array}\right.
\end{aligned}
$$

Thus, the operator $A(u, \cdot): \mathbb{W}_{p}(\Omega) \rightarrow\left(\mathbb{W}_{p}(\Omega)\right)^{*}$ is pseudo-monotone for each $u \in \mathfrak{A}_{a d}$. Hence, following the well-know existence result (see, for instance, [28] [29]), there exists at least one solution $y=y(u)$ of vari- 
ational inequality (61) such that $y \in K_{\varepsilon}$.

As an obvious consequence of Lemma 3, we have the following noteworthy property of penalized OCP (63) (64).

Corollary 3 For each $\varepsilon \in(0, \delta)$ the feasible set $\hat{\Xi}_{\varepsilon}$ is nonempty.

To proceed further, we introduce the following notion.

Definition 6.1. An operator $A: \mathfrak{A}_{a d} \times \mathbb{W}_{p}(\Omega) \rightarrow\left(\mathbb{W}_{p}(\Omega)\right)^{*}$ is said to be quasi-monotone if for any sequence $\left\{\left(u_{k}, y_{k}\right)\right\}_{k=1}^{\infty}$ such that $\left\{u_{k}\right\}_{k \in \mathbb{N}} \subset \mathfrak{A}_{a d}$ and $\left(u_{k}, y_{k}\right) \stackrel{\tau}{\longrightarrow}(u, y)$ in $B V(\Omega) \times W_{0}^{2, p}\left(\Omega ; \Gamma_{D}\right)$, the condition

$$
\limsup _{k \rightarrow \infty}\left\langle A\left(u_{k}, y_{k}\right), y_{k}-y\right\rangle_{\left(\mathbb{W}_{p}(\Omega)\right)^{*} ; \mathbb{W}_{p}(\Omega)} \leq 0
$$

implies the relation

$$
\langle A(u, y), y-v\rangle_{\left(\mathbb{W}_{p}(\Omega)\right)^{*} ; \mathbb{W}_{p}(\Omega)} \leq \liminf _{k \rightarrow \infty}\left\langle A\left(u_{k}, y_{k}\right), y_{k}-v\right\rangle_{\left(\mathbb{W}_{p}(\Omega)\right)^{*} ; \mathbb{W}_{p}(\Omega)}
$$

for all $v \in W_{0}^{2, p}\left(\Omega ; \Gamma_{D}\right)$.

Definition 6.2. We say that an operator $A: \mathfrak{A}_{a d} \times \mathbb{W}_{p}(\Omega) \rightarrow\left(\mathbb{W}_{p}(\Omega)\right)^{*}$ possesses the property $(\mathfrak{M})$, if for any sequence $\left\{\left(u_{k}, y_{k}\right)\right\}_{k=1}^{\infty}$ such that $\left\{u_{k}\right\}_{k \in \mathbb{N}} \subset \mathfrak{A}_{a d}$ and $\left(u_{k}, y_{k}\right) \stackrel{\tau}{\longrightarrow}(u, y)$ in $B V(\Omega) \times W_{0}^{2, p}\left(\Omega ; \Gamma_{D}\right)$, the conditions

$$
A\left(u_{k}, y_{k}\right) \rightarrow d \text { in }\left(\mathbb{W}_{p}(\Omega)\right)^{*}, \quad \limsup _{k \rightarrow \infty}\left\langle A\left(u_{k}, y_{k}\right), y_{k}\right\rangle_{\left(\mathbb{W}_{p}(\Omega)\right)^{*} ; \mathbb{W}_{p}(\Omega)} \leq\langle d, y\rangle_{\left(\mathbb{W}_{p}(\Omega)\right)^{*} ; \mathbb{W}_{p}(\Omega)}
$$

imply the relation $d=A(u, y)$.

Our next intention is to prove the following crucial result.

Theorem 10. The operator $A: \mathfrak{A}_{a d} \times \mathbb{W}_{p}(\Omega) \rightarrow\left(\mathbb{W}_{p}(\Omega)\right)^{*}$, given by formula (29), is quasi-monotone provided assumptions (11)-(14) hold true.

Proof. Let $\left\{\left(u_{k}, y_{k}\right)\right\}_{k=1}^{\infty}$ be a sequence such that $\left\{u_{k}\right\}_{k \in \mathbb{N}} \subset \mathfrak{A}_{a d}$ and $\left(u_{k}, y_{k}\right) \stackrel{\tau}{\longrightarrow}(u, y)$ in

$B V(\Omega) \times W_{0}^{2, p}\left(\Omega ; \Gamma_{D}\right)$. We assume that inequality (67) holds true. Our aim is to establish the relation (68). With that in mind, we set

$$
\begin{aligned}
& \langle B(u, v, y), w\rangle_{\left(\mathbb{W}_{p}(\Omega)\right)^{*} ; \mathbb{W}_{p}(\Omega)}:=\int_{\Omega} u|\Delta y|^{p-2} \Delta y \Delta w \mathrm{~d} x+\int_{\Omega} F(x, v) w \mathrm{~d} x, \\
& \forall u \in \mathfrak{A}_{a d} \text { and } \forall y, v, w \in W_{0}^{2, p}\left(\Omega ; \Gamma_{D}\right),
\end{aligned}
$$

and divide our proof onto several steps.

Step 1. We show that, for each $v \in W_{0}^{2, p}\left(\Omega ; \Gamma_{D}\right)$,

$$
\begin{aligned}
& \lim _{k \rightarrow \infty}\left\langle B\left(u_{k}, y_{k}, v\right), y_{k}-y\right\rangle_{\left(\mathbb{W}_{p}(\Omega)\right)^{*} ; \mathbb{W}_{p}(\Omega)} \\
& :=\lim _{k \rightarrow \infty} \int_{\Omega} u_{k}|\Delta v|^{p-2} \Delta v\left(\Delta y_{k}-\Delta y\right) \mathrm{d} x+\lim _{k \rightarrow \infty} \int_{\Omega} F\left(x, y_{k}\right)\left(y_{k}-y\right) \mathrm{d} x=0 .
\end{aligned}
$$

Indeed, since $y_{k} \rightarrow y$ in $W_{0}^{2, p}\left(\Omega ; \Gamma_{D}\right)$, it follows by the Sobolev embedding theorem that $y_{k} \rightarrow y$ in $L^{s}(\Omega)$ for all $s \in\left(1, p^{*}\right)$. Hence, making use of the subcritical growth condition (12), we get

$$
\begin{aligned}
\int_{\Omega} F\left(x, y_{k}\right)\left(y_{k}-y\right) \mathrm{d} x & \leq C_{1} \int_{\Omega}\left|y_{k}\right|^{r-1}\left|y_{k}-y\right| \mathrm{d} x \\
& \leq C_{1} \sup _{k \in \mathbb{N}}\left\|y_{k}\right\|_{L^{r}(\Omega)}^{r-1}\left\|y_{k}-y\right\|_{L^{r}(\Omega)} \rightarrow 0 \quad \text { as } k \rightarrow \infty .
\end{aligned}
$$

As for the first term in (70), we note that $u_{k} \rightarrow u$ in $L^{r}(\Omega)$ for every $1 \leq r<+\infty$, because $u, u_{k} \in L^{\infty}(\Omega)$ for all $k \in \mathbb{N}$ by the initial assumptions. Hence,

$$
\int_{\Omega}|\Delta v|^{p}\left|u_{k}-u\right|^{q} \mathrm{~d} x \rightarrow 0, \quad \forall v \in W_{0}^{2, p}\left(\Omega ; \Gamma_{D}\right)
$$

by the Lebesgue Dominated Theorem. Since the sequence $\left\{\zeta_{k}:=u_{k}|\Delta v|^{p-2} \Delta v\right\}_{k \in \mathbb{N}}$ is bounded in $L^{q}(\Omega)$ and 


$$
\left\|\zeta_{k}-u|\Delta v|^{p-2} \Delta v\right\|_{L^{q}(\Omega)}^{q}=\int_{\Omega}|\Delta v|^{p}\left|u_{k}-u\right|^{q} \mathrm{~d} x,
$$

it follows from (72) that $u_{k}|\Delta v|^{p-2} \Delta v \rightarrow u|\Delta v|^{p-2} \Delta v$ strongly in $L^{q}(\Omega)$. Therefore, the first term in (70) tends to zero as $k \rightarrow \infty$ as the product of strongly and weakly convergent sequences. Combining this fact with (71), we arrive at the desired property (70).

Step 2. Let us show that

$$
\begin{aligned}
& \lim _{k \rightarrow \infty}\left\langle B\left(u_{k}, y_{k}, v\right), w\right\rangle_{\left(\mathbb{W}_{p}(\Omega)\right)^{*} ; \mathbb{W}_{p}(\Omega)} \\
& =\lim _{k \rightarrow \infty} \int_{\Omega} u_{k}|\Delta v|^{p-2} \Delta v \Delta w \mathrm{~d} x+\lim _{k \rightarrow \infty} \int_{\Omega} F\left(x, y_{k}\right) w \mathrm{~d} x \\
& =\int_{\Omega} u|\Delta v|^{p-2} \Delta v \Delta w \mathrm{~d} x+\int_{\Omega} F(x, y) w \mathrm{~d} x \\
& =\langle B(u, y, v), w\rangle_{\left(\mathbb{W}_{p}(\Omega)\right)^{*} ; \mathbb{W}_{p}(\Omega)}, \quad \forall v, w \in W_{0}^{2, p}\left(\Omega ; \Gamma_{D}\right) .
\end{aligned}
$$

By analogy with the previous step, we note that $u_{k} \rightarrow u$ in $L^{r}(\Omega)$ for every $1 \leq r<+\infty$. In particular, this yields $u_{k} \varphi \rightarrow u \varphi$ strongly in $L^{q}(\Omega) \quad \forall \varphi \in L^{\infty}(\Omega)$. In view of this, we infer

$$
\lim _{k \rightarrow \infty} \int_{\Omega} u_{k} \Delta w \varphi \mathrm{d} x=\int_{\Omega} u \Delta w \varphi \mathrm{d} x \quad \forall \varphi \in L^{\infty}(\Omega), \forall w \in W_{0}^{2, p}\left(\Omega ; \Gamma_{D}\right) .
$$

This means that

$$
u_{k} \Delta w \rightarrow u \Delta w \text { in } L^{1}(\Omega) .
$$

But we also have that the sequence $\left\{u_{k} \Delta w\right\}_{k \in \mathbb{N}}$ is bounded in $L^{p}(\Omega)$. Hence, $u_{k} \Delta w \rightarrow u \Delta w$ in $L^{p}(\Omega)$ for each $w \in W_{0}^{2, p}\left(\Omega ; \Gamma_{D}\right)$. Since $|\Delta v|^{p-2} \Delta v \in L^{q}(\Omega)$ for any $v \in W_{0}^{2, p}\left(\Omega ; \Gamma_{D}\right)$, it follows that

$$
\lim _{k \rightarrow \infty} \int_{\Omega} u_{k} \Delta w|\Delta v|^{p-2} \Delta v \mathrm{~d} x=\int_{\Omega} u \Delta w|\Delta v|^{p-2} \Delta v \mathrm{~d} x
$$

by definition of the weak convergence in $L^{p}(\Omega)$. Thus, in order to conclude the equality (73), it remains to show that

$$
\lim _{k \rightarrow \infty} \int_{\Omega} F\left(x, y_{k}\right) w \mathrm{~d} x=\int_{\Omega} F(x, y) w \mathrm{~d} x, \quad \forall w \in W_{0}^{2, p}\left(\Omega ; \Gamma_{D}\right) .
$$

In view of the subcritical growth condition (12), we have the following estimate

$$
\begin{aligned}
\|\left. F\left(\cdot, y_{k}\right)\right|_{L^{r-1}(\Omega)} ^{r /(r-1)} & :=\int_{\Omega}\left|F\left(x, y_{k}\right)\right|^{\frac{r}{r-1}} \mathrm{~d} x \leq \int_{\Omega}\left(C_{1}\left|y_{k}\right|^{r-1}\right)^{\frac{r}{r-1}} \mathrm{~d} x \\
& \leq C_{1}^{r /(r-1)}\left\|y_{k}\right\|_{L^{r}(\Omega)}^{r} \leq C_{1}^{r /(r-1)} N_{*}^{r} \sup _{k \in \mathbb{N}}\left\|y_{k}\right\|_{p . \Delta},
\end{aligned}
$$

where $N_{*}$ is the norm of the embedding operator $W^{2, p}(\Omega) \hookrightarrow L^{r}(\Omega)$. Hence, we may suppose that the sequence $\left\{F\left(x, y_{k}\right)\right\}_{k \in \mathbb{N}}$ is compact with respect to the weak convergence in $\frac{r}{r-1}(\Omega)$ and, therefore, there exists an element $\psi \in L^{\frac{r}{r-1}}(\Omega)$ such that, up to a subsequence,

$$
F\left(x, y_{k}\right) \rightarrow \psi \text { in } \frac{r}{r-1}(\Omega) \text { as } k \rightarrow \infty .
$$

Thus, to conclude this step, we have to show that $\psi=F(x, y)$. By monotonicity property (13), it follows that for every $z \in \mathbb{R}$ and every positive function $\phi \in C_{0}^{\infty}(\Omega)$, we have

$$
\int_{\Omega} \phi(x)\left(F\left(x, y_{k}\right)-F(x, z)\right)\left(y_{k}-z\right) \mathrm{d} x \geq 0 .
$$

So, taking into account (76) and the fact that $y_{k} \rightarrow y$ strongly in $L^{r}(\Omega)$ by Sobolev embedding theorem, we can pass to the limit in this inequality as $k \rightarrow \infty$. As a result, we get 


$$
\int_{\Omega} \phi(x)(\psi-F(x, z))(y-z) \mathrm{d} x \geq 0, \quad \forall z \in \mathbb{R}
$$

for all positive $\phi \in C_{0}^{\infty}(\Omega)$. After localization, we have

$$
(\psi-F(x, z))(y-z) \geq 0, \quad \forall z \in \mathbb{R} .
$$

Since the function $F: \Omega \times \mathbb{R} \rightarrow \mathbb{R}$ is strictly monotone, it follows that $\psi=F(x, y)$. Thus, the relation (75) is a direct consequence of the convergence (76).

Step 3. This is the final step of our proof. As follows from (69), for every element $v \in W_{0}^{2, p}\left(\Omega ; \Gamma_{D}\right)$ and each index $k \in \mathbb{N}$, we have the estimate

$$
\begin{aligned}
& \left\langle B\left(u_{k}, y_{k}, y_{k}\right)-B\left(u_{k}, y_{k}, v\right), y_{k}-v\right\rangle_{\left(\mathbb{W}_{p}(\Omega)\right)^{*} ; \mathbb{W}_{p}(\Omega)} \\
& :=\int_{\Omega} u_{k}\left(\left|\Delta y_{k}\right|^{p-2} \Delta y_{k}-|\Delta v|^{p-2} \Delta v\right)\left(\Delta y_{k}-\Delta v\right) \mathrm{d} x \\
& \geq \alpha \int_{\Omega} 2^{2-p}\left|\Delta y_{k}-\Delta v\right|^{p} \mathrm{~d} x>0, \quad \forall y_{k} \neq v \text { in } \Omega .
\end{aligned}
$$

Let $v \in W_{0}^{2, p}\left(\Omega ; \Gamma_{D}\right)$ be a fixed element. We put $y_{\sigma}=(1-\sigma) y+\sigma v$ for all $\sigma \in[0,1]$. Taking into account the monotonicity condition (77), we see that

$$
\left\langle B\left(u_{k}, y_{k}, y_{k}\right)-B\left(u_{k}, y_{k}, y_{\sigma}\right), y_{k}-y_{\sigma}\right\rangle_{\left(\mathbb{W}_{p}(\Omega)\right)^{*} ; \mathbb{W}_{p}(\Omega)} \geq 0 .
$$

Since $A(u, y)=B(u, y, y)$, it follows from (78) that

$$
\begin{aligned}
& \sigma\left\langle A\left(u_{k}, y_{k}\right), y-v\right\rangle_{\left(\mathbb{W}_{p}(\Omega)\right)^{*} ; \mathbb{W}_{p}(\Omega)} \\
& \geq-\left\langle A\left(u_{k}, y_{k}\right), y_{k}-y\right\rangle_{\left(\mathbb{W}_{p}(\Omega)\right)^{*} ; \mathbb{W}_{p}(\Omega)}+\left\langle B\left(u_{k}, y_{k}, y_{\sigma}\right), y_{k}-y\right\rangle_{\left(\mathbb{W}_{p}(\Omega)\right)^{*} ; \mathbb{W}_{p}(\Omega)} \\
& \quad+\sigma\left\langle B\left(u_{k}, y_{k}, y_{\sigma}\right), y-v\right\rangle_{\left(\mathbb{W}_{p}(\Omega)\right)^{*} ; \mathbb{W}_{p}(\Omega)} .
\end{aligned}
$$

Passing to the limit in (79) as $k \rightarrow \infty$, we obtain

$$
\begin{aligned}
& \sigma \liminf _{k \rightarrow \infty}\left\langle A\left(u_{k}, y_{k}\right), y-v\right\rangle_{\left(\mathbb{W}_{p}(\Omega)\right)^{*} ; \mathbb{W}_{p}(\Omega)} \\
& \geq-\limsup \left\langle A\left(u_{k}, y_{k}\right), y_{k}-y\right\rangle_{\left(\mathbb{W}_{p}(\Omega)\right)^{*} ; \mathbb{W}_{p}(\Omega)} \\
& +\liminf _{k \rightarrow \infty}\left\langle B\left(u_{k}, y_{k}, y_{\sigma}\right), y_{k}-y\right\rangle_{\left(\mathbb{W}_{p}(\Omega)\right)^{*} ; \mathbb{W}_{p}(\Omega)} \\
& +\sigma \liminf _{k \rightarrow \infty}\left\langle B\left(u_{k}, y_{k}, y_{\sigma}\right), y-v\right\rangle_{\left(\mathbb{W}_{p}(\Omega)\right)^{*} ; \mathbb{W}_{p}(\Omega)},
\end{aligned}
$$

where

$$
\begin{aligned}
& \liminf _{k \rightarrow \infty}\left\langle B\left(u_{k}, y_{k}, y_{\sigma}\right), y_{k}-y\right\rangle_{\left(\mathbb{W}_{p}(\Omega)\right)^{*} ; \mathbb{W}_{p}(\Omega)} \\
& =\lim _{k \rightarrow \infty}\left\langle B\left(u_{k}, y_{k}, y_{\sigma}\right), y_{k}-y\right\rangle_{\left(\mathbb{W}_{p}(\Omega)\right)^{*} ; \mathbb{W}_{p}(\Omega)} \stackrel{\text { by }(70)}{=} 0, \\
& \limsup _{k \rightarrow \infty}\left\langle A\left(u_{k}, y_{k}\right), y_{k}-y\right\rangle_{\left(\mathbb{W}_{p}(\Omega)\right)^{*} ; \mathbb{W}_{p}(\Omega)} \stackrel{\text { by (67) }}{\geq} 0,
\end{aligned}
$$

and

$$
\lim _{k \rightarrow \infty}\left\langle B\left(u_{k}, y_{k}, y_{\sigma}\right), y-v\right\rangle_{\left(\mathbb{W}_{p}(\Omega)\right)^{*} ; \mathbb{W}_{p}(\Omega)} \stackrel{\text { by (73) }}{=}\left\langle B\left(u, y, y_{\sigma}\right), y-v\right\rangle_{\left(\mathbb{W}_{p}(\Omega)\right)^{*} ; \mathbb{W}_{p}(\Omega)} .
$$

Hence, for each $\sigma \in[0,1]$, we have the inequality

$$
\liminf _{k \rightarrow \infty}\left\langle A\left(u_{k}, y_{k}\right), y-v\right\rangle_{\left(\mathbb{W}_{p}(\Omega)\right)^{*} ; \mathbb{W}_{p}(\Omega)} \geq\left\langle B\left(u, y, y_{\sigma}\right), y-v\right\rangle_{\left(\mathbb{W}_{p}(\Omega)\right)^{*} ; \mathbb{W}_{p}(\Omega)} .
$$


Since the convergence $y_{\sigma} \rightarrow y$ is strong in $W_{0}^{2, p}\left(\Omega ; \Gamma_{D}\right)$, it follows that $\left|\Delta y_{\sigma}\right|^{p-2} \Delta y_{\sigma} \rightarrow|\Delta y|^{p-2} \Delta y$ strongly in $L^{q}(\Omega)$, and therefore,

$$
\begin{aligned}
& \left\langle B\left(u, y, y_{\sigma}\right), y-v\right\rangle_{\left(\mathbb{W}_{p}(\Omega)\right)^{*} ; \mathbb{W}_{p}(\Omega)} \\
& \stackrel{\sigma \rightarrow 0}{\longrightarrow} \int_{\Omega} u|\Delta y|^{p-2} \Delta y(\Delta y-\Delta v) \mathrm{d} x+\int_{\Omega} F(x, y)(y-v) \mathrm{d} x=:\langle A(u, y), y-v\rangle_{\left(\mathbb{W}_{p}(\Omega)\right)^{*} ; \mathbb{W}_{p}(\Omega)} .
\end{aligned}
$$

As a result, we deduce from (81) and (82) that

$$
\begin{aligned}
& \liminf _{k \rightarrow \infty}\left\langle A\left(u_{k}, y_{k}\right), y_{k}-v\right\rangle_{\left(\mathbb{W}_{p}(\Omega)\right)^{*} ; \mathbb{W}_{p}(\Omega)} \\
& \geq \liminf _{k \rightarrow \infty}\left\langle A\left(u_{k}, y_{k}\right), y_{k}-y\right\rangle_{\left(\mathbb{W}_{p}(\Omega)\right)^{*} ; \mathbb{W}_{p}(\Omega)}+\liminf _{k \rightarrow \infty}\left\langle A\left(u_{k}, y_{k}\right), y-v\right\rangle_{\left(\mathbb{W}_{p}(\Omega)\right)^{*} ; \mathbb{W}_{p}(\Omega)} \\
& \geq \liminf _{k \rightarrow \infty}\left\langle A\left(u_{k}, y_{k}\right), y_{k}-y\right\rangle_{\left(\mathbb{W}_{p}(\Omega)\right)^{*} ; \mathbb{W}_{p}(\Omega)}+\langle A(u, y), y-v\rangle_{\left(\mathbb{W}_{p}(\Omega)\right)^{*} ; \mathbb{W}_{p}(\Omega)} \\
& \geq \liminf _{k \rightarrow \infty}\left\langle B\left(u_{k}, y_{k}, y_{k}\right)-B\left(u_{k}, y_{k}, y\right), y_{k}-y\right\rangle_{\left(\mathbb{W}_{p}(\Omega)\right)^{*} ; \mathbb{W}_{p}(\Omega)} \\
& \quad+\liminf _{k \rightarrow \infty}\left\langle B\left(u_{k}, y_{k}, y\right), y_{k}-y\right\rangle_{\left(\mathbb{W}_{p}(\Omega)\right)^{*} ; \mathbb{W}_{p}(\Omega)}+\langle A(u, y), y-v\rangle_{\left(\mathbb{W}_{p}(\Omega)\right)^{*} ; \mathbb{W}_{p}(\Omega)} \\
& \quad \stackrel{\text { by (78) }}{\geq} \liminf _{k \rightarrow \infty}\left\langle B\left(u_{k}, y_{k}, y\right), y_{k}-y\right\rangle_{\left(\mathbb{W}_{p}(\Omega)\right)^{*} ; \mathbb{W}_{p}(\Omega)}+\langle A(u, y), y-v\rangle_{\left(\mathbb{W}_{p}(\Omega)\right)^{*} ; \mathbb{W}_{p}(\Omega)} \\
& \quad \text { by (70) }\langle A(u, y), y-v\rangle_{\left(\mathbb{W}_{p}(\Omega)\right)^{*} ; \mathbb{W}_{p}(\Omega)},
\end{aligned}
$$

that is, the inequality (68) is valid.

Remark 6.1. In fact (see [19], Remark 3.13), we have the following implication:

" $A$ is quasi-monotone" $\Rightarrow$ " $A$ possesses the property $(\mathfrak{M}) . "$

Hence, in view of Theorem 10, we can claim that the operator $A: \mathfrak{A}_{a d} \times \mathbb{W}_{p}(\Omega) \rightarrow\left(\mathbb{W}_{p}(\Omega)\right)^{*}$, which is defined by relation (29), possesses the property $(\mathfrak{M})$.

We are now in a position to show that the penalized optimal control problem in the coefficient of variational inequality (63)-(64) is solvable for each value $\varepsilon \in(0, \delta)$.

Lemma 4 If the assumptions (11)-(14) are valid, then the OCP (63)-(64) admits at least one solution $\left(u_{\varepsilon}^{0}, y_{\varepsilon}^{0}\right) \in \hat{\Xi}_{\varepsilon}$ for every fixed $\varepsilon \in(0, \delta)$ and any $f \in W^{-2, q}\left(\Omega ; \Gamma_{D}\right), \quad y_{d} \in L^{2}(\Omega)$, and $\zeta^{\max } \in L^{p}(\partial \Omega)$.

Proof. Let $\left\{\left(u_{k}, y_{k}\right)\right\}_{k=1}^{\infty} \subset U_{\partial} \times K$ be a minimizing sequence to problem (63)-(64). The coerciveness property (66) and estimate

$$
\begin{aligned}
\left\langle A\left(u_{k}, y_{k}\right), y_{k}-\zeta\right\rangle_{\left(\mathbb{W}_{p}(\Omega)\right)^{*} ; \mathbb{W}_{p}(\Omega)} & \leq\left\langle f, y_{k}-\zeta\right\rangle_{\left(\mathbb{W}_{p}(\Omega)\right)^{*} ; \mathbb{W}_{p}(\Omega)} \\
& \leq\|f\|_{W^{-2, q}\left(\Omega ; \Gamma_{D}\right)}\left\|y_{k}-\zeta\right\|_{p, \Delta}
\end{aligned}
$$

immediately imply that the sequence $\left\{y_{k}\right\}_{k=1}^{\infty}$ is bounded in $W_{0}^{2, p}\left(\Omega ; \Gamma_{D}\right)$. Indeed, using the notations $V=\mathbb{W}_{p}(\Omega)$ and $V^{*}=\left(\mathbb{W}_{p}(\Omega)\right)^{*}$, we have

$$
\frac{\langle A y, y-\zeta\rangle_{V^{*} ; V}}{\|y-\zeta\|_{V}} \geq \frac{\langle A y, y-\zeta\rangle_{V^{*} ; V}}{\|y\|_{V}+\|\zeta\|_{V}}=\frac{\langle A y, y-\zeta\rangle_{V^{*} ; V}}{\|y\|_{V}} \cdot \frac{1}{1+\frac{\|\zeta\|_{V}}{\|y\|_{V}}} \rightarrow+\infty \text { as }\|y\|_{V} \rightarrow \infty .
$$

On the other hand, from (83) it follows that

$$
\frac{\langle A y, y-\zeta\rangle_{V^{*} ; V}}{\|y-\zeta\|_{V}} \leq \frac{\langle f, y-\zeta\rangle_{V^{*} ; V}}{\|y-\zeta\|_{V}} \leq\|f\|_{V^{*}}=\|f\|_{W^{-2, q}\left(\Omega ; \Gamma_{D}\right)} .
$$

So, comparing these two chains of relations, we arrive at the existence of a constant $C>0$ such that $C$ is independent of $u \in \mathfrak{A}_{a d}$ and $\|y\|_{V} \leq C$ as far as $y \in K_{\varepsilon}$ is a solution to (63).

Since 


$$
\sup _{k \in \mathbb{N}} \int_{\Omega}\left|D u_{k}\right| \leq \sup _{k \in \mathbb{N}} \hat{I}_{\varepsilon}\left(u_{k}, y_{k}\right)<+\infty
$$

and the set $\mathfrak{A}_{a d} \times K_{\varepsilon}$ is sequentially closed with respect to the $\tau$-convergence, we may assume by Theroem 1 that there exists a pair $\left(u_{\varepsilon}^{0}, y_{\varepsilon}^{0}\right) \in \mathfrak{A}_{a d} \times K_{\varepsilon}$ such that $\left(u_{k}, y_{k}\right) \stackrel{\tau}{\longrightarrow}\left(u_{\varepsilon}^{0}, y_{\varepsilon}^{0}\right)$. Then passing to the limit in

$$
\left\langle A\left(u_{k}, y_{k}\right), \zeta-y_{k}\right\rangle_{\left(\mathbb{W}_{p}(\Omega)\right)^{*} ; \mathbb{W}_{p}(\Omega)} \geq\left\langle f, \zeta-y_{k}\right\rangle_{\left(\mathbb{W}_{p}(\Omega)\right)^{*} ; \mathbb{W}_{p}(\Omega)}
$$

as $k \rightarrow \infty$, we obtain

$$
\limsup _{k \rightarrow \infty}\left\langle A\left(u_{k}, y_{k}\right), y_{k}-\zeta\right\rangle_{\left(\mathbb{W}_{p}(\Omega)\right)^{*} ; \mathbb{W}_{p}(\Omega)} \leq\left\langle f, \zeta-y_{\varepsilon}^{0}\right\rangle_{\left(\mathbb{W}_{p}(\Omega)\right)^{*} ; \mathbb{W}_{p}(\Omega)}, \quad \forall \zeta \in K_{\varepsilon} .
$$

Having put here $\zeta=y_{\varepsilon}^{0} \in K_{\varepsilon}$, we arrive at the inequality

$$
\limsup _{k \rightarrow \infty}\left\langle A\left(u_{k}, y_{k}\right), y_{k}-y_{\varepsilon}^{0}\right\rangle_{\left(\mathbb{W}_{p}(\Omega)\right)^{*} ; \mathbb{W}_{p}(\Omega)} \leq 0 .
$$

Hence,

$$
\liminf _{k \rightarrow \infty}\left\langle A\left(u_{k}, y_{k}\right), y_{k}-\zeta\right\rangle_{\left(\mathbb{W}_{p}(\Omega)\right)^{*} ; \mathbb{W}_{p}(\Omega)} \geq\left\langle A\left(u_{\varepsilon}^{0}, y_{\varepsilon}^{0}\right), y_{\varepsilon}^{0}-\zeta\right\rangle_{\left(\mathbb{W}_{p}(\Omega)\right)^{*} ; \mathbb{W}_{p}(\Omega)}, \quad \forall \zeta \in K_{\varepsilon},
$$

by the quasi-monotonicity property of the operator $A$. Combining this inequality with (84), we come to the relation

$$
\left\langle A\left(u_{\varepsilon}^{0}, y_{\varepsilon}^{0}\right), \zeta-y_{\varepsilon}^{0}\right\rangle_{\left(\mathbb{W}_{p}(\Omega)\right)^{*} ; \mathbb{W}_{p}(\Omega)} \geq\left\langle f, \zeta-y_{\varepsilon}^{0}\right\rangle_{\left(\mathbb{W}_{p}(\Omega)\right)^{*} ; \mathbb{W}_{p}(\Omega)} \quad \forall \zeta \in K_{\varepsilon} .
$$

Thus, $\left(u_{\varepsilon}^{0}, y_{\varepsilon}^{0}\right) \in \mathfrak{A}_{a d} \times K_{\varepsilon}$ is a feasible pair to the problem (63)-(64).

Let us show that $\left(u_{\varepsilon}^{0}, y_{\varepsilon}^{0}\right)$ is an optimal pair to this problem. As follows from (83), the sequence $\left\{A\left(u_{k}, y_{k}\right)\right\}_{k \in \mathbb{N}}$ is bounded in $\left(\mathbb{W}_{p}(\Omega)\right)^{*}$. Let $d$ be its weak limit in $\left(\mathbb{W}_{p}(\Omega)\right)^{*}$ as $k \rightarrow \infty$. Then

$$
\begin{aligned}
& \limsup _{k \rightarrow \infty}\left\langle A\left(u_{k}, y_{k}\right), y_{k}\right\rangle_{\left(\mathbb{W}_{p}(\Omega)\right)^{*} ; \mathbb{W}_{p}(\Omega)} \\
& \leq\left\langle f, y_{\varepsilon}^{0}-\zeta\right\rangle_{\left(\mathbb{W}_{p}(\Omega)\right)^{*} ; \mathbb{W}_{p}(\Omega)}+\langle d, \zeta\rangle_{\left(\mathbb{W}_{p}(\Omega)\right)^{*} ; \mathbb{W}_{p}(\Omega)} \\
& =\left\langle d, y_{\varepsilon}^{0}\right\rangle>_{\left(\mathbb{W}_{p}(\Omega)\right)^{*} ; \mathbb{W}_{p}(\Omega)}+\left\langle d-f, \zeta-y_{\varepsilon}^{0}\right\rangle_{\left(\mathbb{W}_{p}(\Omega)\right)^{*} ; \mathbb{W}_{p}(\Omega)} \quad \forall \zeta \in K_{\varepsilon} .
\end{aligned}
$$

Substituting $y_{\varepsilon}^{0}$ for $\zeta$ in the last inequality, we get

$$
\limsup _{k \rightarrow \infty}\left\langle A\left(u_{k}, y_{k}\right), y_{k}\right\rangle_{\left(\mathbb{W}_{p}(\Omega)\right)^{*} ; \mathbb{W}_{p}(\Omega)} \leq\left\langle d, y_{\varepsilon}^{0}\right\rangle_{\left(\mathbb{W}_{p}(\Omega)\right)^{*} ; \mathbb{W}_{p}(\Omega)} .
$$

Since the quasi-monotone operator possesses the $(\mathfrak{M})$-property (see Remark 6.6), it follows that $d=A\left(u_{\varepsilon}^{0}, y_{\varepsilon}^{0}\right)$. As a result, using the $\tau$-lower semicontinuity property of the cost functional (63), we finally obtain

$$
\inf _{(u, y) \in \hat{\bar{\Xi}}_{\varepsilon}} \hat{I}_{\varepsilon}(u, y)=\liminf _{k \rightarrow \infty} \hat{I}_{\varepsilon}\left(u_{k}, y_{k}\right) \geq I\left(u_{\varepsilon}^{0}, y_{\varepsilon}^{0}\right)+\varepsilon^{-1}\left\|A\left(u_{\varepsilon}^{0}, y_{\varepsilon}^{0}\right)-f\right\|_{\left(\mathbb{W}_{p}(\Omega)\right)^{*}}=\hat{I}_{\varepsilon}\left(u_{\varepsilon}^{0}, y_{\varepsilon}^{0}\right) .
$$

Thus, $\left(u_{\varepsilon}^{0}, y_{\varepsilon}^{0}\right)$ is an optimal pair to the penalized problem (63)-(64).

The next step of our analysis is to consider a sequence of optimal pairs $\left\{\left(u_{\varepsilon}^{0}, y_{\varepsilon}^{0}\right)\right\}_{\varepsilon>0} \subset \mathfrak{A}_{a d} \times K_{\varepsilon}$ in the limit as $\varepsilon$ tends to 0 .

Theorem 11. Let $\left\{\left(u_{\varepsilon}^{0}, y_{\varepsilon}^{0}\right)\right\}_{\varepsilon>0}$ be a sequence of optimal pairs to penalized problems (63) - (64). In addition to the assumptions of Lemma 4 , assume that there exists a constant $C>0$ such that

$$
\sup _{\varepsilon>0} \hat{I}_{\varepsilon}\left(u_{\varepsilon}^{0}, y_{\varepsilon}^{0}\right) \leq C \text {. }
$$

Then the sequence $\left\{\left(u_{\varepsilon}^{0}, y_{\varepsilon}^{0}\right)\right\}$ is relatively compact with respect to the $\tau$-convergence and each of its $\tau$-cluster pair $\left(u^{0}, y^{0}\right)$ is such thiat (up to a subsequence)

$$
\begin{gathered}
\left(u_{\varepsilon}^{0}, y_{\varepsilon}^{0}\right) \stackrel{\tau}{\longrightarrow}\left(u^{0}, y^{0}\right) \text { as } \varepsilon \rightarrow 0, \\
\left(u^{0}, y^{0}\right) \in \Xi, \text { and } I\left(u^{0}, y^{0}\right)=\inf _{(u, y) \in \Xi} I(u, y),
\end{gathered}
$$


i.e. $\left(u^{0}, y^{0}\right)$ is an optimal pair to the original OCP (19).

Proof. Let $\left\{\left(u_{\varepsilon}^{0}, y_{\varepsilon}^{0}\right)\right\}_{\varepsilon>0}$ be a given sequence of optimal pairs to penalized problems (63)-(64). Since each of the set $K_{\varepsilon} \subset W_{0}^{2, p}\left(\Omega ; \Gamma_{D}\right)$ contains zero, we have

$$
\begin{aligned}
\alpha\|y\|_{W_{0}^{2, p}\left(\Omega ; \Gamma_{D}\right)}^{p} & \leq \int_{\Omega} u_{\varepsilon}^{0}\left|\Delta y_{\varepsilon}^{0}\right|^{p} \mathrm{~d} x=\left\langle A\left(u_{\varepsilon}^{0}, y_{\varepsilon}^{0}\right), y_{\varepsilon}^{0}\right\rangle_{\left(\mathbb{W}_{p}(\Omega)\right)^{*} ; \mathbb{W}_{p}(\Omega)} \\
& \quad \leq \quad\left\langle f, y_{\varepsilon}^{0}\right\rangle_{\left(\mathbb{W}_{p}(\Omega)\right)^{*} ; \mathbb{W}_{p}(\Omega)} \leq\|f\|_{W^{-2, q}\left(\Omega ; \Gamma_{D}\right)}\left\|y_{\varepsilon}^{0}\right\|_{p, \Delta} .
\end{aligned}
$$

Hence, the following estimate for the optimal states takes place

$$
\left\|y_{\varepsilon}^{0}\right\|_{p, \Delta} \leq\left(\alpha^{-1}\|f\|_{W^{-2, q}\left(\Omega ; \Gamma_{D}\right)}\right)^{\frac{1}{p-1}}, \quad \forall u \in \mathfrak{A}_{a d} .
$$

Let us show that the sequence of corresponding optimal controls $\left\{u_{\varepsilon}^{0}\right\}$ is $B V$-bounded. Indeed, due to the estimate (85), the numerical sequence $\left\{\hat{I}_{\varepsilon}\left(u_{\varepsilon}^{0}, y_{\varepsilon}^{0}\right)\right\}$ is uniformly bounded with respect to $\varepsilon$. Hence, in view of the structure of the cost functional (63), we deduce

$$
\left\|A\left(u_{\varepsilon}^{0}, y_{\varepsilon}^{0}\right)-f\right\|_{\mathbb{Y}^{*}} \leq \varepsilon C, \quad \int_{\Omega}\left|D u_{\varepsilon}^{0}\right| \leq C .
$$

From this, we immediately conclude that $\sup _{\varepsilon>0}\left\|u_{\varepsilon}^{0}\right\|_{B V(\Omega)}<+\infty$, and, hence, due to Theorem 1, Proposition 7, and estimate (88), we may assume that there exists a pair $\left(u^{0}, y^{0}\right) \in \mathfrak{A}_{a d} \times K$ such that $\left(u_{\varepsilon}^{0}, y_{\varepsilon}^{0}\right) \stackrel{\tau}{\longrightarrow}\left(u^{0}, y^{0}\right)$ as $\varepsilon \rightarrow 0$ in $B V(\Omega) \times W_{0}^{2, p}\left(\Omega ; \Gamma_{D}\right)$ (here, we have used the fact that the sets $K_{\varepsilon}$ converge in Kuratowski sense to $K$, see the proof of Theorem 8).

Let us show that the pair $\left(u^{0}, y^{0}\right)$ is feasible to the original problem (19). Using the arguments of the proof of Lemma 4, we have $A\left(u_{\varepsilon}^{0}, y_{\varepsilon}^{0}\right) \rightarrow d$ in $\left(\mathbb{W}_{p}(\Omega)\right)^{*}$ and $d=A\left(u^{0}, y^{0}\right)$. Then, as follows from (89), we have

$$
0 \leq\left\|A\left(u^{0}, y^{0}\right)-f\right\|_{\left(\mathbb{W}_{p}(\Omega)\right)^{*}} \leq \liminf _{\varepsilon \rightarrow 0}\left\|A\left(u_{\varepsilon}^{0}, y_{\varepsilon}^{0}\right)-f\right\|_{\left(\mathbb{W}_{p}(\Omega)\right)^{*}}=\lim _{\varepsilon \rightarrow 0}\left\|A\left(u_{\varepsilon}^{0}, y_{\varepsilon}^{0}\right)-f\right\|_{\left(\mathbb{W}_{p}(\Omega)\right)^{*}}=0 .
$$

Thus, $A\left(u^{0}, y^{0}\right)=f$ as elements of $\left(\mathbb{W}_{p}(\Omega)\right)^{*}$ and, hence, $\left(u^{0}, y^{0}\right) \in \Xi$.

It remains to prove that $\left(u^{0}, y^{0}\right)$ is an optimal pair. If, on the contrary, we assume that the exists a pair $\left(u^{*}, y^{*}\right) \in \Xi$ such that $I\left(u^{*}, y^{*}\right)<I\left(u^{0}, y^{0}\right)$, then

$$
I\left(u_{\varepsilon}^{0}, y_{\varepsilon}^{0}\right) \leq I\left(u_{\varepsilon}^{0}, y_{\varepsilon}^{0}\right)+\varepsilon^{-1}\left\|A\left(u_{\varepsilon}^{0}, y_{\varepsilon}^{0}\right)-f\right\|_{\left(\mathbb{W}_{p}(\Omega)\right)^{*}} \leq I\left(u^{*}, y^{*}\right), \quad \forall \varepsilon>0 .
$$

Therefore, passing to the limit in this inequality as $\varepsilon \rightarrow 0$ and using the w-lower semicontinuity property of the cost functional, we finally get

$$
I\left(u^{0}, y^{0}\right) \leq \liminf _{\varepsilon \rightarrow 0} I\left(u_{\varepsilon}^{0}, y_{\varepsilon}^{0}\right) \leq I\left(u^{*}, y^{*}\right) .
$$

This contradiction immediately leads us to the conclusion: The $\left(u^{0}, y^{0}\right)$ is an optimal pair to the OCP (19).

Remark 6.2. As follows from the proof of Theorem 11, whatever the sequence of optimal solutions $\left\{\left(u_{\varepsilon}^{0}, y_{\varepsilon}^{0}\right)\right\}$ to the penalized problems (63)-(64) has been chosen, if this sequence satisfies condition (85), then it always gives in the limit as $\varepsilon \rightarrow 0$ some optimal pair to the original OCP (19). However, it is unknown whether the entire set of the solutions to OCP (19) can be attained in such way.

Remark 6.3. It is easy to see that in the case if the feasible set to the original OCP is nonempty, it suffices to guarantee the fulfilment of assumption (85). Indeed, let $(u, y) \in \Xi$ be any feasible pair to the original OCP (19). Then $\hat{I}_{\varepsilon}(u, y)=I(u, y)$ for each $\varepsilon>0$. Since $\left(u_{\varepsilon}^{0}, y_{\varepsilon}^{0}\right)$ is an optimal pair to problem (63)-(64), this yields

$$
\sup _{\varepsilon>0} \hat{I}_{\varepsilon}\left(u_{\varepsilon}^{0}, y_{\varepsilon}^{0}\right) \leq \sup _{\varepsilon>0} \hat{I}_{\varepsilon}(u, y)=I(u, y)
$$

and we arrive at the inequality (85). 


\section{Acknowledgements}

Research is funded by DFG-Excellence Cluster Engineering for Advanced Materials.

\section{References}

[1] Sprekels, J. and Tiba, D. (2005) Optimal Design of Mechanical Structures. In: Imanuvilov, O., Leugering, G., Triggiani, R., et al., Eds., Control Theory of Partial Differential Equations, Chapman\& Hall/CRC, Boca Raton, 259- 271.

[2] Sprekels, J. and Tiba, D. (2009) Optimization Problems for Thin Elastic Structures. Number 158 in International Series of Numerical Mathematics, Birkhauser Verlag, Basel.

[3] Neitaanmaki, P., Sprekles, J. and Tiba, D. (2006) Optimization of Elliptic Systems, Theory and Applications. Springer Monographs in Mathematics, Springer-Verlag, Berlin.

[4] Gazzola, F., Grunau, H.-C. and Sweers, G. (2010) Polyharmonic Boundary Value Problems: Positivity Preserving and Nonlinear Higher Order Elliptic Equations in Bounded Domains. Springer-Verlag, Berlin. http://dx.doi.org/10.1007/978-3-642-12245-3

[5] Litvinov, V.G. (1987) Optimization in Elliptic Boundary Value Problems with Applications to Machanics. MIR, Moscow.

[6] Lurie, K.A. (1993) Applied Optimal Control Theory of Distributed Systems. Planum Press.

[7] Khan, A. and Sama, M. (2013) A New Conical Regularization for Some Optimization and Optimal Control Problems: Convergence Analysis and Finite Element Discretization. Numerical Functional Analysis and Optimization, 34, 861895. http://dx.doi.org/10.1080/01630563.2013.806546

[8] Adams, R. (1975) Sobolv Spaces. Academic Press, Cambridge.

[9] Lions, J.-L. and Magenes, E. (1968) Problemes aux limites non homogenes et applications. Vol. 1, Dunod, Paris.

[10] Bucur, D. and Gazzola, F. (2011) The First Biharmonic Steklov Eigenvalue: Positivity Preserving and Shape Optimization. Milan Journal of Mathematics, 79, 247-258. http://dx.doi.org/10.1007/s00032-011-0143-x

[11] Gilbarg, D. and Trudinger, N.S. (2001) Elliptic Partial Differential Equations of Second Order. Springer-Verlag, Berlin.

[12] Colasuonno, F. and Pucci, P. (2001) Multiplicity of Solutions for p(x)-Polyharmonic Elliptic Kirchoff Equations. Nonlinear Analysis, Theory, Methods, and Applications, 74, 5962-5974. http://dx.doi.org/10.1016/j.na.2011.05.073

[13] Lubyshev, V.F. (2011) Multiple Solutions of an Even-Order Nonlinear Problems with Convex-Concave Nonlinearity. Nonlinear Analysis, Theory, Methods, and Applications, 74, 1345-1354. http://dx.doi.org/10.1016/j.na.2010.10.007

[14] Giusti, E. (1984) Minimal Surfaces and Functions of Bounded Variation. Birkhauser Verlag, Basel. http://dx.doi.org/10.1007/978-1-4684-9486-0

[15] Attouch, H., Buttazzo, G. and Michaille, G. (2006) Variational Analysis in Sobolev and BV Spaces: Application to PDE and Optimization. SIAM, Philadelphia.

[16] Roubicek, T. (2013) Nonlinear Partial Differential Equations with Applications. Birkhauser Verlag, Basel. http://dx.doi.org/10.1007/978-3-0348-0513-1

[17] Lions, J.-L. (1969) Some Methods of Solving Non-Linear Boundary Value Problems. Dunod-Gauthier-Villars, Paris.

[18] Casas, E. (1992) Optimal Control in the Coefficients of Elliptic Equations with State Constraints. Applied Mathematics \& Optimization, 26, 21-37. http://dx.doi.org/10.1007/BF01218394

[19] Kogut, P.I. and Leugering, G. (2011) Optimal Control Problems for Partial Differential Equations on Reticulated Domains: Approximation and Asymptotic Analysis, Birkhauser Verlag, Basel. http://dx.doi.org/10.1007/978-0-8176-8149-4

[20] Roubicek, T. (1997) Relaxation in Optimization Theory and Variational Calculus. De Gruyter, Berlin, New York. http://dx.doi.org/10.1515/9783110811919

[21] Borwein, J.M. and Zhuang, D.M. (1993) Super Efficiency in Vector Optimization. Transactions of the American Mathematical Society, 338, 105-122. http://dx.doi.org/10.1090/S0002-9947-1993-1098432-5

[22] Henig, M.I. (1982) Proper Efficiency with Respect to Cones. Journal of Optimization Theory and Applications, 36, 387-407. http://dx.doi.org/10.1007/BF00934353

[23] Makarov, E.K. and Rackkovski, N.N. (1999) Unified Representation of Proper Efficiency by Means of Dilating Cones. Journal of Optimization Theory and Applications, 101, 141-165. http://dx.doi.org/10.1023/A:1021775112119

[24] Zhuang, D.M. (1994) Density Result for Proper Efficiencies. SIAM Journal on Control and Optimization, 32, 51-58. http://dx.doi.org/10.1137/S0363012989171518 
[25] Jahn, J. (2004) Vector Optimization: Theory, Applications, and Extensions. Springer-Verlag, Berlin. http://dx.doi.org/10.1007/978-3-540-24828-6

[26] Schiel, R. (2014) Vector Optimization and Control with PDEs and Pointwise State Constraints. Phd Thesis, Friedrich-Alexander-University Erlangen-Nurnberg, Nurnberg.

[27] Mel'nik, V.S. (1986) Method of Monotone Operators in the Theory of Constrained Optimal System. Rep. Ukrain. Acad. Sci., 7, 64-67.

[28] Barbu, V. (1993) Analysis and Control of Infinite Dimensional Systems. Academic Press, Cambridge.

[29] Ekeland, I. and Temam, R. (1976) Convex Analysis and Variational Problems. Elsevier, Amsterdam.

Submit or recommend next manuscript to SCIRP and we will provide best service for you:

Accepting pre-submission inquiries through Email, Facebook, LinkedIn, Twitter, etc.

A wide selection of journals (inclusive of 9 subjects, more than 200 journals)

Providing 24-hour high-quality service

User-friendly online submission system

Fair and swift peer-review system

Efficient typesetting and proofreading procedure

Display of the result of downloads and visits, as well as the number of cited articles

Maximum dissemination of your research work

Submit your manuscript at: http://papersubmission.scirp.org/ 